December, 2012

\title{
Conformal supergravities as Chern-Simons theories revisited
}

\author{
Sergei M. Kuzenko and Gabriele Tartaglino-Mazzucchellit \\ School of Physics M013, The University of Western Australia \\ 35 Stirling Highway, Crawley W.A. 6009, Australia
}

\begin{abstract}
We propose a superfield method to construct off-shell actions for $\mathcal{N}$-extended conformal supergravity theories in three space-time dimensions. It makes use of the superform technique to engineer supersymmetric invariants. The method is specifically applied to the case of $\mathcal{N}=1$ conformal supergravity and provides a new realization for the actions of conformal and topologically massive supergravities.
\end{abstract}

\footnotetext{
${ }^{1}$ gabriele.tartaglino-mazzucchelli@uwa.edu.au
} 


\section{Introduction}

Inspired by the construction of three-dimensional $\mathcal{N}=1$ topologically massive supergravity [1, 2], conformal supergravities in three dimensions were formulated as supersymmetric Chern-Simons theories for $\mathcal{N}=1[3], \mathcal{N}=2$ [4], and finally for arbitrary $\mathcal{N}$ [5]. The approaches pursued in [3, 4, 5] are purely component. They are on-shell for $\mathcal{N}>2$, and do not allow for a conventional superspace reformulation for $\mathcal{N}>1$. The action for $\mathcal{N}=1$ conformal supergravity can readily be constructed in terms of the superfield connection as a superspace integral [6, 7, 8] (although the results in [6, 17, 8] are incomplete, see section 3). However, such a construction becomes impossible starting from $\mathcal{N}=2$. This is because (i) the spinor and vector sectors of the superfield connection have positive dimension equal to $1 / 2$ and 1 respectively; and (ii) the dimension of the full superspace measure is $(\mathcal{N}-3)$. As a result, it is not possible to construct contributions to the action that are cubic in the superfield connection for $\mathcal{N} \geq 2$.

Nevertheless, it turns out that $\mathcal{N}$-extended conformal supergravity can be realized in terms of the off-shell Weyl supermultiplet [9] and the associated curved superspace geometry originally sketched in [9] and later fully developed in [10]. Such a realization is a generalization of the superform formulation for the linear multiplet in four-dimensional $\mathcal{N}=2$ conformal supergravity given in [11].1 In the present paper we will first describe our method by explicitly constructing a new action principle for $\mathcal{N}=1$ conformal supergravity in three dimensions. After doing so, an outline will be given as to how this method should be used in the case of extended conformal supergravities.

Three-dimensional $\mathcal{N}=1$ supergravity is an old topic that goes back to 1977 . Without pretending to give a complete literature review, here we only list several works [15, 16, 17] which initiated this research topic.

This paper is organized as follows. In section 2 we review the superspace geometry of $\mathcal{N}=1$ conformal supergravity. In section 3 we describe standard actions for conformal supergravity and topologically massive supergravity realized as superspace integrals. Here we also present the $\mathcal{N}=1$ supersymmetric Cotton tensor. Section 4 describes our main construction. A component analysis of our results is given in section 5 . In section 6 we give a sketch of our method as applied to extended conformal supergravity. The main body of the paper is accompanied by two technical appendices concerning a locally supersymmetric action and exact three-forms in superspace.

\footnotetext{
${ }^{1}$ At the heart of the formulation [11] lies the superform approach to the construction of supersymmetric invariants [12, 13, 14], also know as the ectoplasm formalism [13, 14].
} 


\section{$2 \mathcal{N}=1$ conformal supergravity in superspace}

In this section we review the geometry of $\mathcal{N}=1$ conformal supergravity in superspace following the notation and conventions of [10]. Let us consider a curved superspace, which is locally parametrized by real bosonic $\left(x^{m}\right)$ and real fermionic $\left(\theta^{\mu}\right)$ coordinates

$$
z^{M}=\left(x^{m}, \theta^{\mu}\right), \quad m=0,1,2, \quad \mu=1,2 .
$$

The superspace structure group is the double covering of the Lorentz group, $\mathrm{SL}(2, \mathbb{R})$, and we denote by $\mathcal{M}_{a b}=-\mathcal{M}_{b a}$ the Lorentz generators. The covariant derivatives have the form:

$$
\mathcal{D}_{A}=\left(\mathcal{D}_{a}, \mathcal{D}_{\alpha}\right)=E_{A}+\Omega_{A}
$$

Here the vector fields $E_{A}=E_{A}^{M} \partial / \partial z^{M}$ define the inverse vielbein, and

$$
\Omega_{A}=\frac{1}{2} \Omega_{A}^{b c} \mathcal{M}_{b c}=-\Omega_{A}{ }^{b} \mathcal{M}_{b}=\frac{1}{2} \Omega_{A}^{\beta \gamma} \mathcal{M}_{\beta \gamma}, \quad \mathcal{M}_{a b}=-\mathcal{M}_{b a}, \quad \mathcal{M}_{\alpha \beta}=\mathcal{M}_{\beta \alpha}
$$

is the Lorentz connection. The Lorentz generators with two vector indices $\left(\mathcal{M}_{a b}\right)$, with one vector index $\left(\mathcal{M}_{a}\right)$ and with two spinor indices $\left(\mathcal{M}_{\alpha \beta}\right)$ are related to each other by the rules: $\mathcal{M}_{a}=\frac{1}{2} \varepsilon_{a b c} \mathcal{M}^{b c}$ and $\mathcal{M}_{\alpha \beta}=\left(\gamma^{a}\right)_{\alpha \beta} \mathcal{M}_{a}$.

The supergravity gauge group is generated by local transformations of the form

$$
\delta_{\mathcal{K}} \mathcal{D}_{A}=\left[\mathcal{K}, \mathcal{D}_{A}\right], \quad \mathcal{K}=\xi^{C} E_{C}+\frac{1}{2} K^{c d} \mathcal{M}_{c d}
$$

with all the gauge parameters obeying natural reality conditions but otherwise arbitrary. Given a tensor superfield $T$, it transforms as follows:

$$
\delta_{\mathcal{K}} T=\mathcal{K} T
$$

The covariant derivatives satisfy the (anti)commutation relations

$$
\left[\mathcal{D}_{A}, \mathcal{D}_{B}\right\}=T_{A B}{ }^{C} \mathcal{D}_{C}+\frac{1}{2} R_{A B}{ }^{c d} \mathcal{M}_{c d}
$$

with $T_{A B}{ }^{C}$ the torsion and $R_{A B}{ }^{c d}$ the Lorentz curvature. Similar to the connection, the Lorentz curvature can be realized in three different forms as tensors carrying two vector indices $\left(R_{A B}{ }^{c d}\right)$, one vector index $\left(R_{A B}^{c}\right)$ and two spinor indices $\left(R_{A B}^{\gamma \delta}\right)$. These are related to each other by the rules: $R_{A B}{ }^{c}=\frac{1}{2} \varepsilon^{c d e} R_{A B d e}$ and $R_{A B}{ }^{\gamma \delta}=\left(\gamma_{c}\right)^{\gamma \delta} R_{A B}{ }^{c}$. 
To describe conformal supergravity, the covariant derivatives have to obey certain constraints such that the complete algebra of covariant derivatives, compatible with all the Bianchi identities, is

$$
\begin{aligned}
\left\{\mathcal{D}_{\alpha}, \mathcal{D}_{\beta}\right\} & =2 \mathrm{i} \mathcal{D}_{\alpha \beta}-4 \mathrm{i} \mathcal{S} \mathcal{M}_{\alpha \beta}, \\
{\left[\mathcal{D}_{a}, \mathcal{D}_{\beta}\right]=} & \mathcal{S}\left(\gamma_{a}\right)_{\beta}{ }^{\gamma} \mathcal{D}_{\gamma}-\left(\gamma_{a}\right)_{\beta}{ }^{\gamma} C_{\gamma \delta \rho} \mathcal{M}^{\delta \rho}-\frac{2}{3}\left(\left(\mathcal{D}_{\beta} \mathcal{S}\right) \delta_{a}^{c}-2 \varepsilon_{a b}{ }^{c}\left(\gamma^{b}\right)_{\beta \gamma}\left(\mathcal{D}^{\gamma} \mathcal{S}\right)\right) \mathcal{M}_{c}, \\
{\left[\mathcal{D}_{a}, \mathcal{D}_{b}\right]=} & \varepsilon_{a b c}\left\{\left[\frac{\mathrm{i}}{2}\left(\gamma^{c}\right)_{\alpha \beta} C^{\alpha \beta \gamma}-\frac{2 \mathrm{i}}{3}\left(\gamma^{c}\right)^{\beta \gamma}\left(\mathcal{D}_{\beta} \mathcal{S}\right)\right] \mathcal{D}_{\gamma}\right. \\
& \left.\quad-\left[-\frac{\mathrm{i}}{2}\left(\gamma^{c}\right)^{\alpha \beta}\left(\gamma^{d}\right)^{\gamma \delta} \mathcal{D}_{(\alpha} C_{\beta \gamma \delta)}-\left(\frac{2 \mathrm{i}}{3}\left(\mathcal{D}^{2} \mathcal{S}\right)+4 \mathcal{S}^{2}\right) \eta^{c d}\right] \mathcal{M}_{d}\right\}
\end{aligned}
$$

Here the scalar $\mathcal{S}$ is real and the spinor $C_{\alpha \beta \gamma}=C_{(\alpha \beta \gamma)}$ is real and completely symmetric and $\mathcal{D}^{2}:=\mathcal{D}^{\alpha} \mathcal{D}_{\alpha}$. The dimension-2 Bianchi identities imply that

$$
\mathcal{D}_{\alpha} C_{\beta \gamma \delta}=\mathcal{D}_{(\alpha} C_{\beta \gamma \delta)}-\mathrm{i} \varepsilon_{\alpha(\beta} \mathcal{D}_{\gamma \delta)} \mathcal{S},
$$

and hence

$$
\mathcal{D}^{\gamma} C_{\alpha \beta \gamma}=-\frac{4 \mathrm{i}}{3} \mathcal{D}_{\alpha \beta} \mathcal{S} .
$$

The algebra of covariant derivatives is invariant under arbitrary super-Weyl transformations [7, 8, 18] of the form

$$
\begin{aligned}
\delta_{\sigma} \mathcal{D}_{\alpha} & =\frac{1}{2} \sigma \mathcal{D}_{\alpha}+\left(\mathcal{D}^{\beta} \sigma\right) \mathcal{M}_{\alpha \beta}, \\
\delta_{\sigma} \mathcal{D}_{a} & =\sigma \mathcal{D}_{a}+\frac{\mathrm{i}}{2}\left(\gamma_{a}\right)^{\gamma \delta}\left(\mathcal{D}_{\gamma} \sigma\right) \mathcal{D}_{\delta}+\varepsilon_{a b c}\left(\mathcal{D}^{b} \sigma\right) \mathcal{M}^{c},
\end{aligned}
$$

with the parameter $\sigma$ being a real unconstrained superfield, provided the torsion superfields transform as

$$
\begin{aligned}
\delta_{\sigma} \mathcal{S} & =\sigma \mathcal{S}-\frac{\mathrm{i}}{4} \mathcal{D}^{2} \sigma \\
\delta_{\sigma} C_{\alpha \beta \gamma} & =\frac{3}{2} \sigma C_{\alpha \beta \gamma}-\frac{1}{2} \mathcal{D}_{(\alpha \beta} \mathcal{D}_{\gamma)} \sigma .
\end{aligned}
$$

The super-Weyl invariance is compulsory for the above geometry to describe the multiplet of $\mathcal{N}=1$ conformal supergravity. This local symmetry will play a crucial role in the present paper.

We introduce the vielbein and connection one-forms defined by

$$
E^{A}:=\mathrm{d} z^{M} E_{M}{ }^{A},
$$




$$
\Omega_{C}{ }^{D}:=\mathrm{d} z^{M} \Omega_{M C}{ }^{D}=E^{A} \Omega_{A C}{ }^{D},
$$

where the supermatrix $E_{M}{ }^{A}$ is the inverse of $E_{A}{ }^{M}$,

$$
E_{M}^{A} E_{A}^{N}=\delta_{M}^{N}, \quad E_{A}{ }^{M} E_{M}^{B}=\delta_{A}^{B} .
$$

The connection one-form is associated with the Lorentz group,

$$
\Omega_{A}{ }^{B}=\left(\begin{array}{c|c}
\Omega_{a}^{b} & 0 \\
\hline 0 & \frac{1}{2} \Omega_{\alpha}{ }^{\beta}
\end{array}\right)=\left(\begin{array}{c|c}
-\varepsilon_{d a}{ }^{b} \Omega^{d} & 0 \\
\hline 0 & \frac{1}{2}\left(\gamma_{d}\right)_{\alpha}^{\beta} \Omega^{d}
\end{array}\right) .
$$

The superspace geometry of $\mathcal{N}=1$ conformal supergravity can be recast in terms of superforms which will be a crucial ingredient of our construction. The torsion and the curvature two-forms are

$$
\begin{aligned}
T^{C} & :=\frac{1}{2} E^{B} \wedge E^{A} T_{A B}{ }^{C}=-\mathrm{d} E^{C}+E^{B} \wedge \Omega_{B}{ }^{C}, \\
R_{C}{ }^{D} & :=\frac{1}{2} E^{B} \wedge E^{A} R_{A B C}{ }^{D}=\mathrm{d} \Omega_{C}{ }^{D}-\Omega_{C}{ }^{E} \wedge \Omega_{E}{ }^{D} .
\end{aligned}
$$

Here we have explicitly indicated the operation of wedge product $(\wedge)$ of superforms. However, in the remainder of the paper it will be assumed and not be given explicitly. Given a $p$-form $F_{p}:=\frac{1}{p !} \mathrm{d} z^{M_{p}} \cdots \mathrm{d} z^{M_{1}} F_{M_{1} \cdots M_{p}}$ its exterior derivative can be written in two different forms:

$$
\begin{aligned}
\mathrm{d} F_{p} & =\frac{1}{p !} \mathrm{d} z^{M_{p}} \cdots \mathrm{d} z^{M_{1}} \mathrm{~d} z^{N} \partial_{N} F_{M_{1} \cdots M_{p}} \\
& =\frac{1}{p !} E^{A_{p}} \cdots E^{A_{1}} E^{B}\left\{\mathcal{D}_{B} F_{A_{1} \cdots A_{p}}-\frac{p}{2} T_{B A_{1}}{ }^{C} F_{C A_{2} \cdots A_{p}}\right\} .
\end{aligned}
$$

For the subsequent analysis we will need the super-Weyl transformations of the vielbein and the connection one-forms. They are

$$
\begin{aligned}
\delta_{\sigma} E^{\alpha} & =-\frac{1}{2} \sigma E^{\alpha}-\frac{\mathrm{i}}{2}\left(\gamma_{b}\right)^{\alpha \beta}\left(\mathcal{D}_{\beta} \sigma\right) E^{b}, \\
\delta_{\sigma} E^{a} & =-\sigma E^{a},
\end{aligned}
$$

and

$$
\delta_{\sigma} \Omega^{c}=E^{\alpha}\left\{-\left(\gamma^{c}\right)_{\alpha \beta}\left(\mathcal{D}^{\beta} \sigma\right)\right\}+E^{a}\left\{-\varepsilon_{a b}^{c}\left(\mathcal{D}^{b} \sigma\right)\right\} .
$$

To construct a locally supersymmetric action principle, we need a real scalar Lagrangian $\mathcal{L}$, of mass dimension +2 , with the super-Weyl transformation law [10]

$$
\delta_{\sigma} \mathcal{L}=2 \sigma \mathcal{L}
$$


The action is

$$
S=\mathrm{i} \int \mathrm{d}^{3} x \mathrm{~d}^{2} \theta E \mathcal{L}, \quad E^{-1}=\operatorname{Ber}\left(E_{A}{ }^{M}\right)
$$

The action is super-Weyl invariant, since the super-Weyl transformation of $E$ proves to be $\delta_{\sigma} E=-2 \sigma E$. Instead of defining the action principle using the superspace integration, it suffices to construct a three-form $\Xi(\mathcal{L})$ which is constructed in terms of $\mathcal{L}$ and possesses the following properties: (i) $\Xi(\mathcal{L})$ is closed, $\mathrm{d} \Xi(\mathcal{L})=0$; (ii) $\Xi(\mathcal{L})$ is super-Weyl invariant, $\delta_{\sigma} \Xi(\mathcal{L})=0$; (iii) $\Xi(\mathcal{L})$ is dimensionless. In fact, the conditions (ii) and (iii) prove to completely determine (modulo an overall factor) the explicit form of $\Xi(\mathcal{L})$ to be

$$
\begin{aligned}
\Xi(\mathcal{L})= & \frac{\mathrm{i}}{2} E^{\gamma} E^{\beta} E^{a}\left(\gamma_{a}\right)_{\beta \gamma} \mathcal{L}+\frac{1}{4} E^{\gamma} E^{b} E^{a} \varepsilon_{a b c}\left(\gamma^{c}\right)_{\gamma}{ }^{\delta} \mathcal{D}_{\delta} \mathcal{L} \\
& -\frac{1}{24} E^{c} E^{b} E^{a} \varepsilon_{a b c}\left(\mathrm{i} \mathcal{D}^{2}+8 \mathcal{S}\right) \mathcal{L}
\end{aligned}
$$

It is a simple exercise to check that this form is closed, $\mathrm{d} \Xi(\mathcal{L})=0$. This three-form coincides with that originally constructed in [20] by directly solving the cohomological problem d $\Xi=0$.

\section{Traditional approach to conformal supergravity and topologically massive supergravity}

With the matrix notation $\Omega_{A}=\left(\Omega_{A \beta}{ }^{\gamma}\right)$, the action for conformal supergravity is

$$
\begin{aligned}
S_{\mathrm{CSG}}= & \int \mathrm{d}^{3} x \mathrm{~d}^{2} \theta E \Omega^{\alpha \beta \gamma}\left\{C_{\alpha \beta \gamma}-\frac{4}{3} \varepsilon_{\alpha(\beta} \mathcal{D}_{\gamma)} \mathcal{S}\right\} \\
& -\frac{1}{3} \int \mathrm{d}^{3} x \mathrm{~d}^{2} \theta E\left\{\operatorname{tr}\left(\frac{1}{4} \Omega^{\alpha} \Omega^{\beta} \Omega_{\alpha \beta}+2 \mathcal{S} \Omega^{\alpha} \Omega_{\alpha}\right)+\mathcal{S} \Omega_{\alpha \beta}^{\alpha \beta} \Omega_{\beta \gamma}^{\gamma}\right\} \\
& +16 \mathrm{i} \int \mathrm{d}^{3} x \mathrm{~d}^{2} \theta E \mathcal{S}^{2} .
\end{aligned}
$$

The tensor in the braces in the first line of 3.1 is divergenceless with respect to its first index,

$$
\mathcal{D}^{\alpha}\left\{C_{\alpha \beta \gamma}-\frac{4}{3} \varepsilon_{\alpha(\beta} \mathcal{D}_{\gamma)} \mathcal{S}\right\}=0
$$

Modulo an overall coefficient, the structures in the first and second lines of (3.1) are uniquely fixed by the condition of invariance under the local Lorentz transformations

$$
\delta_{K} \Omega_{A}^{b c}=K_{A}^{D} \Omega_{D}^{b c}-\mathcal{D}_{A} K^{b c} .
$$


The last term in (3.1) is uniquely fixed by requiring invariance under the super-Weyl transformations

$$
\begin{aligned}
\delta_{\sigma} \Omega_{\alpha}{ }^{\gamma \delta} & =\frac{1}{2} \sigma \Omega_{\alpha}{ }^{\gamma \delta}+2 \delta_{\alpha}^{(\gamma} \mathcal{D}^{\delta)} \sigma \\
\delta_{\sigma} \Omega_{\alpha \beta}{ }^{\gamma \delta} & =\sigma \Omega_{\alpha \beta}{ }^{\gamma \delta}-\mathrm{i}\left(\mathcal{D}_{(\alpha} \sigma\right) \Omega_{\beta)}{ }^{\gamma \delta}+2 \delta_{(\alpha}^{(\gamma} \mathcal{D}_{\beta)}^{\delta)} \sigma
\end{aligned}
$$

The topological mass term appearing in the first two lines of 3.1 was given in Superspace [6], eq. (2.6.47). However, since $3 \mathrm{D} \mathcal{N}=1$ super-Weyl invariance was not discussed in [6], the complete action (3.1) was not presented in this book. Instead the requirement of super-Weyl invariance was put forward by Zupnik and Pak [7, 8, who derived an action of the form (3.1). However, their completely symmetric torsion $C_{\alpha \beta \gamma}$ was chosen to obey the constraint $\mathcal{D}^{\alpha} C_{\alpha \beta \gamma}=0$ which holds only in a special super-Weyl gauge such that $\mathcal{D}_{\alpha \beta} \mathcal{S}=0$, due to the Bianchi identity 2.9 . So the actual status of the action presented in [7, 8] is not quite clear to us.

Varying the conformal supergravity action (3.1) with respect to a supergravity prepotential [6] leads to the $\mathcal{N}=1$ supersymmetric Cotton tensor ${ }^{2}$

$$
W_{\alpha \beta \gamma}=\frac{\mathrm{i}}{2} \mathcal{D}^{2} C_{\alpha \beta \gamma}+\mathcal{D}_{(\alpha \beta} \mathcal{D}_{\gamma)} \mathcal{S}+4 \mathcal{S} C_{\alpha \beta \gamma}
$$

It is an instructive exercise to prove that the super-Weyl transformation of $W_{\alpha \beta \gamma}$ is

$$
\delta_{\sigma} W_{\alpha \beta \gamma}=\frac{5}{2} \sigma W_{\alpha \beta \gamma}
$$

The equation of motion for conformal supergravity is

$$
W_{\alpha \beta \gamma}=0
$$

At the same time, this equation is the necessary and sufficient condition for the superspace to be conformally flat.

The action for three-dimensional $\mathcal{N}=1$ Poincaré supergravity is

$$
S_{\mathrm{PSG}}=-\mathrm{i} \int \mathrm{d}^{3} x \mathrm{~d}^{2} \theta E\left\{\frac{\mathrm{i}}{2} \mathcal{D}^{\alpha} \varphi \mathcal{D}_{\alpha} \varphi-\mathcal{S} \varphi^{2}+\frac{\lambda}{4} \varphi^{4}\right\},
$$

with $\lambda$ a cosmological constant. This action is super-Weyl invariant provided $\varphi$ transforms by the rule

$$
\delta_{\sigma} \varphi=\frac{1}{2} \sigma \varphi
$$

\footnotetext{
${ }^{2}$ This expression may be compared with the $\mathcal{N}=2$ supersymmetric Cotton tensor [19].
} 
The scalar field $\varphi$, which is chosen to be nowhere vanishing, plays the role of the conformal compensator. The super-Weyl invariance can be used to impose the gauge $\varphi=1$.

The equations of motion corresponding to the action $(3.8)$ are

$$
\begin{aligned}
\varphi \mathcal{D}_{(\alpha} \mathcal{D}_{\beta \gamma)} \varphi-3 \mathcal{D}_{(\alpha} \varphi \mathcal{D}_{\beta \gamma)} \varphi+\varphi^{2} C_{\alpha \beta \gamma} & =0 \\
\left(\mathrm{i} \mathcal{D}^{2}+2 \mathcal{S}\right) \varphi-\lambda \varphi^{3} & =0
\end{aligned}
$$

Eq. 3.10a is obtained by varying the action with respect to the supergravity prepotential. The left-hand side of (3.10a) is proportional to the supercurrent for the scalar superfield $\varphi$ described by the action (3.8). It is an instructive exercise to show that both equations (3.10a and (3.10b) are super-Weyl invariant.

To describe topologically massive supergravity, we have to consider a linear combination of the two actions (3.1) and (3.8). Then the conformal supergravity equation (3.7) turns into

$$
W_{\alpha \beta \gamma}-m\left(\varphi \mathcal{D}_{(\alpha} \mathcal{D}_{\beta \gamma)} \varphi-3 \mathcal{D}_{(\alpha} \varphi \mathcal{D}_{\beta \gamma)} \varphi+\varphi^{2} C_{\alpha \beta \gamma}\right)=0
$$

with $m$ a parameter of unit mass dimension.

If we choose the super-Weyl gauge $\varphi=1$ and linearize eq. (3.11) around Minkowski superspace, we end up with

$$
\left(\frac{\mathrm{i}}{2} D^{2}-m\right) C_{\alpha \beta \gamma}=0 \quad \Longrightarrow \quad\left(\square-m^{2}\right) C_{\alpha \beta \gamma}=0
$$

with $D^{2}=D^{\alpha} D_{\alpha}$ and $D_{\alpha}$ the spinor derivatives in Minkowski superspace.

\section{Main construction}

In this section we describe the key points of our method as applied to $\mathcal{N}=1$ conformal supergravity.

\subsection{One-parameter family of covariant derivatives}

Of special importance for our analysis is the observation that the geometry of conformal supergravity, which was reviewed in section 2, can be described by a one-parameter family of covariant derivatives $\nabla_{A}$,

$$
\mathcal{D}_{A}=E_{A}+\Omega_{A} \quad \longrightarrow \quad \nabla_{A}=E_{A}+\Omega_{A}
$$


defined as follows

$$
\nabla_{\alpha}=\mathcal{D}_{\alpha}, \quad \nabla_{a}=\mathcal{D}_{a}+2 \lambda \mathcal{S} \mathcal{M}_{a}
$$

with $\lambda$ a real parameter. Only the vector connection becomes deformed,

$$
\boldsymbol{\Omega}_{\alpha}{ }^{c}=\Omega_{\alpha}{ }^{c}, \quad \boldsymbol{\Omega}_{a}{ }^{c}=\Omega_{a}{ }^{c}-2 \lambda \mathcal{S} \delta_{a}{ }^{c} .
$$

We will use boldface notation for the torsion and curvature tensors associated with the deformed covariant derivatives $\nabla_{A}$,

$$
\left[\nabla_{A}, \nabla_{B}\right\}=\boldsymbol{T}_{A B}{ }^{C} \nabla_{C}+\frac{1}{2} \boldsymbol{R}_{A B}{ }^{c d} \mathcal{M}_{c d} .
$$

The algebra of the deformed covariant derivatives is as follows:

$$
\begin{aligned}
\left\{\nabla_{\alpha}, \nabla_{\beta}\right\}= & 2 \mathrm{i} \nabla_{\alpha \beta}-4 \mathrm{i}(1+\lambda) \mathcal{S}\left(\gamma^{c}\right)_{\alpha \beta} \mathcal{M}_{c}, \\
{\left[\nabla_{a}, \nabla_{\beta}\right]=} & (1-\lambda) \mathcal{S}\left(\gamma_{a}\right)_{\beta}{ }^{\gamma} \nabla_{\gamma}-\left[\left(\gamma_{a}\right)_{\beta}{ }^{\gamma}\left(\gamma^{c}\right)^{\delta \rho} C_{\gamma \delta \rho}+\frac{2(1+3 \lambda)}{3}\left(\nabla_{\beta} \mathcal{S}\right) \delta_{a}^{c}\right. \\
& \left.-\frac{4}{3} \varepsilon_{a b}{ }^{c}\left(\gamma^{b}\right)_{\beta \gamma}\left(\nabla^{\gamma} \mathcal{S}\right)\right] \mathcal{M}_{c}, \\
{\left[\nabla_{a}, \nabla_{b}\right]=} & 4 \lambda \mathcal{S}_{a b}{ }^{c} \nabla_{c}+\varepsilon_{a b c}\left[\frac{\mathrm{i}}{2}\left(\gamma^{c}\right)_{\alpha \beta} C^{\alpha \beta \gamma} \nabla_{\gamma}-\frac{2 \mathrm{i}}{3}\left(\gamma^{c}\right)^{\beta \gamma}\left(\nabla_{\beta} \mathcal{S}\right)\right] \nabla_{\gamma} \\
& -\varepsilon_{a b c}\left[-\frac{\mathrm{i}}{2}\left(\gamma^{c}\right)^{\alpha \beta}\left(\gamma^{d}\right)^{\gamma \delta} \nabla_{(\alpha} C_{\beta \gamma \delta)}-\eta^{c d}\left(\frac{2 \mathrm{i}}{3}\left(\nabla^{2} \mathcal{S}\right)+4\left(1-\lambda^{2}\right) \mathcal{S}^{2}\right)\right. \\
& \left.+2 \lambda \varepsilon^{c e d}\left(\nabla_{e} \mathcal{S}\right)\right] \mathcal{M}_{d} .
\end{aligned}
$$

The covariant derivatives $\mathcal{D}_{A}$, which were introduced in section 2 , correspond to the choice $\lambda=0$. On the other hand, the choice $\lambda=-1$ corresponds to the covariant derivatives employed in the book [6].

The spinor covariant derivative $\nabla_{\alpha}$ and the torsion tensors $\mathcal{S}$ and $C_{\alpha \beta \gamma}$ are obviously $\lambda$-independent, and their super-Weyl transformations do not change. The super-Weyl transformation of the deformed vector derivatives $\nabla_{a}$ is

$$
\delta_{\sigma} \nabla_{a}=\sigma \nabla_{a}+\frac{\mathrm{i}}{2}\left(\gamma_{a}\right)^{\gamma \delta}\left(\nabla_{\gamma} \sigma\right) \nabla_{\delta}+\varepsilon_{a b c}\left(\nabla^{b} \sigma\right) \mathcal{M}^{c}-\frac{\lambda \mathrm{i}}{2}\left(\nabla^{2} \sigma\right) \mathcal{M}_{a}
$$

From here we read off the super-Weyl transformation of the deformed connection oneform:

$$
\delta_{\sigma} \Omega^{c}=E^{\alpha}\left\{-\left(\gamma^{c}\right)_{\alpha \beta}\left(\nabla^{\beta} \sigma\right)\right\}+E^{a}\left\{-\varepsilon_{a b}^{c}\left(\nabla^{b} \sigma\right)+\frac{\lambda \mathrm{i}}{2} \delta_{a}^{c}\left(\nabla^{2} \sigma\right)\right\} .
$$

This turns into 2.18 for $\lambda=0$. 


\subsection{Curvature squared four-form}

We are interested in the $\lambda$-dependent four-form

$$
\boldsymbol{R}^{2}:=\boldsymbol{R}^{a} \boldsymbol{R}_{a}=\frac{2}{5} \boldsymbol{R}_{A}^{B} \boldsymbol{R}_{B}^{A}
$$

which is closed for any value of $\lambda$,

$$
\mathrm{d} \boldsymbol{R}^{2}=0
$$

The curvature two-form $\boldsymbol{R}^{d}=\frac{1}{2} E^{B} E^{A} \boldsymbol{R}_{A B}{ }^{d}$ has the following components:

$$
\begin{aligned}
& \boldsymbol{R}_{\alpha \beta}{ }^{d}= 4 \mathrm{i}(1+\lambda) \mathcal{S}\left(\gamma^{d}\right)_{\alpha \beta}, \\
& \boldsymbol{R}_{a \beta}{ }^{d}=\left(\gamma_{a}\right)_{\beta}{ }^{\gamma}\left(\gamma^{d}\right)^{\delta \rho} C_{\gamma \delta \rho}+\frac{1}{3}\left(2(1+3 \lambda)\left(\nabla_{\beta} \mathcal{S}\right) \delta_{a}^{d}-4 \varepsilon_{a b}{ }^{d}\left(\gamma^{b}\right)_{\beta \gamma}\left(\nabla^{\gamma} \mathcal{S}\right)\right) \\
& \boldsymbol{R}_{a b}{ }^{d}=\varepsilon_{a b c}\left[-\frac{\mathrm{i}}{2}\left(\gamma^{c}\right)^{\alpha \beta}\left(\gamma^{d}\right)^{\gamma \delta} \nabla_{(\alpha} C_{\beta \gamma \delta)}-\left(\frac{2 \mathrm{i}}{3}\left(\nabla^{2} \mathcal{S}\right)+4\left(1-\lambda^{2}\right) \mathcal{S}^{2}\right) \eta^{c d}\right. \\
&\left.\quad+2 \lambda \varepsilon^{c e d}\left(\nabla_{e} \mathcal{S}\right)\right] .
\end{aligned}
$$

In terms of the curvature two-form, the form $\boldsymbol{R}^{2}$ reads

$$
\begin{aligned}
\boldsymbol{R}^{2}:= & \boldsymbol{R}^{e} \boldsymbol{R}_{e}=\frac{1}{4} E^{D} E^{C} E^{B} E^{A} \boldsymbol{R}_{A B}{ }^{e} \boldsymbol{R}_{C D e} \\
= & \frac{1}{4} E^{\delta} E^{\gamma} E^{\beta} E^{\alpha} \boldsymbol{R}_{\alpha \beta}{ }^{e} \boldsymbol{R}_{\gamma \delta e}+E^{\delta} E^{\gamma} E^{\beta} E^{a} \boldsymbol{R}_{a \beta}{ }^{e} \boldsymbol{R}_{\gamma \delta e} \\
& +\frac{1}{2} E^{\delta} E^{\gamma} E^{b} E^{a} \boldsymbol{R}_{a b}{ }^{e} \boldsymbol{R}_{\gamma \delta e}-E^{\delta} E^{\gamma} E^{b} E^{a} \boldsymbol{R}_{a \gamma}{ }^{e} \boldsymbol{R}_{b \delta e} \\
& +E^{\delta} E^{c} E^{b} E^{a} \boldsymbol{R}_{a b}{ }^{e} \boldsymbol{R}_{c \delta e} .
\end{aligned}
$$

Using this representation and also defining the components of $\boldsymbol{R}^{2}$ as

$$
\begin{aligned}
\boldsymbol{R}^{2}= & \frac{1}{4 !} E^{D} E^{C} E^{B} E^{A}\left(\boldsymbol{R}^{2}\right)_{A B C D} \\
= & \frac{1}{24} E^{\delta} E^{\gamma} E^{\beta} E^{\alpha}\left(\boldsymbol{R}^{2}\right)_{\alpha \beta \gamma \delta}+\frac{1}{6} E^{\delta} E^{\gamma} E^{\beta} E^{a}\left(\boldsymbol{R}^{2}\right)_{a \beta \gamma \delta}+\frac{1}{4} E^{\delta} E^{\gamma} E^{b} E^{a}\left(\boldsymbol{R}^{2}\right)_{a b \gamma \delta} \\
& +\frac{1}{6} E^{\delta} E^{c} E^{b} E^{a}\left(\boldsymbol{R}^{2}\right)_{a b c \delta},
\end{aligned}
$$

direct calculations give

$$
\begin{aligned}
& \left(\boldsymbol{R}^{2}\right)_{\alpha \beta \gamma \delta}=0 \\
& \left(\boldsymbol{R}^{2}\right)_{a \beta \gamma \delta}=-48 \mathrm{i}(1+\lambda) \mathcal{S}\left(\gamma_{a}\right)_{(\beta}{ }^{\rho} C_{\gamma \delta) \rho}-16 \mathrm{i}(1+\lambda)(1-3 \lambda) \mathcal{S}\left(\nabla_{(\beta} \mathcal{S}\right)\left(\gamma_{a}\right)_{\gamma \delta)} \\
& \left(\boldsymbol{R}^{2}\right)_{a b \gamma \delta}=\varepsilon_{a b c}\left\{4\left(\gamma^{c}\right)_{\gamma \delta} C^{\alpha \rho \tau} C_{\alpha \rho \tau}-8\left(\gamma^{c}\right)^{\rho \tau}\left(\nabla_{(\rho} \mathcal{S}\right) C_{\gamma \delta) \tau}-\frac{8}{3}\left(\gamma^{c}\right)_{(\gamma}{ }^{\rho}\left(\nabla^{\tau} \mathcal{S}\right) C_{\delta) \rho \tau}\right.
\end{aligned}
$$




$$
\begin{aligned}
& -8 \lambda\left(\gamma^{c}\right)^{\rho \tau}\left(\nabla_{(\gamma} \mathcal{S}\right) C_{\delta) \rho \tau}-\frac{32 \lambda}{3}\left(\gamma^{c}\right)_{\gamma \delta}\left(\nabla^{\rho} \mathcal{S}\right)\left(\nabla_{\rho} \mathcal{S}\right) \\
& -8(1+\lambda) \mathcal{S}\left[\left(\gamma^{c}\right)^{\rho \tau}\left(\nabla_{(\gamma} C_{\delta \rho \tau)}\right)+\mathrm{i}\left(\gamma^{c}\right)_{\gamma \delta}\left(\frac{2 \mathrm{i}}{3}\left(\nabla^{2} \mathcal{S}\right)+4\left(1-\lambda^{2}\right) \mathcal{S}^{2}\right)\right. \\
& \left.\left.+2 \mathrm{i} \lambda \varepsilon^{c d e}\left(\gamma_{d}\right)_{\gamma \delta}\left(\nabla_{e} \mathcal{S}\right) \mathcal{S}\right]\right\} \\
& \left(\boldsymbol{R}^{2}\right)_{a b c \delta}=\varepsilon_{a b c}\left\{4 \mathrm{i} C^{\gamma \rho \tau} \nabla_{(\delta} C_{\gamma \rho \tau)}-8(1+3 \lambda)\left[\frac{\mathrm{i}}{3}\left(\nabla^{2} \mathcal{S}\right)+2\left(1-\lambda^{2}\right) \mathcal{S}^{2}\right]\left(\nabla_{\delta} \mathcal{S}\right)\right. \\
& \left.-4 \lambda\left(\gamma^{d}\right)^{\rho \tau}\left(\nabla_{d} \mathcal{S}\right) C_{\delta \rho \tau}+\frac{32 \lambda}{3}\left(\gamma^{d}\right)_{\delta \rho}\left(\nabla^{\rho} \mathcal{S}\right)\left(\nabla_{d} \mathcal{S}\right)\right\} .
\end{aligned}
$$

\subsection{Torsion-induced three-form}

Our next task is to look for a three-form $\boldsymbol{\Sigma}_{\mathrm{T}}=\frac{1}{3 !} E^{C} E^{B} E^{A} \boldsymbol{\Sigma}_{A B C}$ such that (i) it obeys the equation

$$
\mathrm{d} \boldsymbol{\Sigma}=\boldsymbol{R}^{2}
$$

and (ii) its components are constructed in terms of the torsion and its covariant derivatives. The equation 4.13 is equivalent to

$$
4 \nabla_{[A} \boldsymbol{\Sigma}_{B C D)}-6 \boldsymbol{T}_{[A B}{ }^{E} \boldsymbol{\Sigma}_{|E| C D)}=\left(\boldsymbol{R}^{2}\right)_{A B C D} .
$$

Under the additional conditions $\boldsymbol{\Sigma}_{\alpha \beta \gamma}=\boldsymbol{\Sigma}_{a \beta \gamma}=0$, it turns out that eq. 4.14 allows us to completely determine all the components of $\boldsymbol{\Sigma}_{A B C}$ in terms of $\left(\boldsymbol{R}^{2}\right)_{A B C D}$. This relies on the fact that $T_{\alpha \beta}{ }^{c}=2 \mathrm{i}\left(\gamma^{c}\right)_{\alpha \beta}$. The presence of this dimensionless torsion allows us to iteratively use (4.14) to express $\boldsymbol{\Sigma}_{A B C}$ in terms of derivatives of lower mass dimension components and $\left(\boldsymbol{R}^{2}\right)_{A B C D}$. This is a superform analogue of Dragon's theorem [21] (see also [22]) and is a crucial ingredient in iteratively solving superspace Bianchi identities. In particular, by using (4.14), it is not difficult to prove that

$$
\begin{aligned}
& \boldsymbol{\Sigma}_{a b \gamma}=\frac{\mathrm{i}}{40} \varepsilon_{a b c}\left[\left(\gamma^{c}\right)_{\gamma}^{\delta}\left(\gamma^{d}\right)^{\rho \tau}\left(\boldsymbol{R}^{2}\right)_{d \delta \rho \tau}+2 \varepsilon^{c d e}\left(\gamma_{d}\right)^{\delta \rho}\left(\boldsymbol{R}^{2}\right)_{e \gamma \delta \rho}\right], \\
& \boldsymbol{\Sigma}_{a b c}=-\frac{\mathrm{i}}{24} \varepsilon_{a b c}\left(\gamma_{d}\right)^{\gamma \delta} \varepsilon^{d e f}\left[2 \nabla_{(\gamma} \boldsymbol{\Sigma}_{e f \delta)}-\left(\boldsymbol{R}^{2}\right)_{e f \gamma \delta}\right] .
\end{aligned}
$$

Using the explicit form for $\left(\boldsymbol{R}^{2}\right)_{A B C D}$ gives

$$
\begin{aligned}
\Sigma_{\mathrm{T}}=\frac{1}{2} E^{\gamma} E^{b} E^{a} \varepsilon_{a b c}\{ & \left.-4(1+\lambda)\left(\gamma^{c}\right)^{\delta \rho} C_{\gamma \delta \rho} \mathcal{S}-\frac{8(1+\lambda)(1-3 \lambda)}{3}\left(\gamma^{c}\right)_{\gamma}{ }^{\delta}\left(\nabla_{\delta} \mathcal{S}\right) \mathcal{S}\right\} \\
+\frac{1}{6} E^{c} E^{b} E^{a} \varepsilon_{a b c}\{ & 2 \mathrm{i} C^{\alpha \beta \gamma} C_{\alpha \beta \gamma}+\frac{4 \mathrm{i}}{3}\left(1-6 \lambda-3 \lambda^{2}\right)\left(\nabla^{\gamma} \mathcal{S}\right) \nabla_{\gamma} \mathcal{S} \\
+ & \left.4 \mathrm{i}(1+\lambda)(1-\lambda)\left(\nabla^{2} \mathcal{S}\right) \mathcal{S}+16(1+\lambda)\left(1-\lambda^{2}\right) \mathcal{S}^{3}\right\} .
\end{aligned}
$$


One can explicitly check that this three-form satisfies 4.13) or equivalently 4.14). The crucial feature of $\boldsymbol{\Sigma}_{\mathrm{T}}$ is that it is constructed only in terms of the torsion tensor.

There is a natural freedom in the choice of $\boldsymbol{\Sigma}_{\mathrm{T}}$ described by

$$
\boldsymbol{\Sigma}_{\mathrm{T}} \rightarrow \widetilde{\Sigma}_{\mathrm{T}}=\boldsymbol{\Sigma}_{\mathrm{T}}+A \Xi\left(S^{2}\right)
$$

where $A$ is a real parameter, and $\Xi(\mathcal{L})$ denotes the closed three-form 2.21 . The specific feature of the three-form $\widetilde{\Sigma}_{\mathrm{T}}$ with $A \neq 0$ is that $\widetilde{\Sigma}_{a \beta \gamma} \neq 0$.

We need to work out how $\boldsymbol{\Sigma}_{\mathrm{T}}$ behaves under the super-Weyl transformations. For this it is necessary to use the transformation rules for the supervielbein $2.17 \mathrm{a}-2.17 \mathrm{~b}$ ) and the torsion superfields $2.11 \mathrm{a}-2.11 \mathrm{~b}$ ) along with the following equations

$$
\begin{aligned}
& \nabla_{\alpha} \nabla^{2} \sigma=-2 \mathrm{i} \nabla_{\alpha \beta} \nabla^{\beta} \sigma+2 \mathrm{i}(1+3 \lambda) \mathcal{S} \nabla_{\alpha} \sigma=-2 \mathrm{i} \nabla^{\beta} \nabla_{\alpha \beta} \sigma+8 \mathrm{i} \mathcal{S} \nabla_{\alpha} \sigma, \\
& \nabla^{2} \nabla^{2} \sigma=-4 \nabla^{a} \nabla_{a} \sigma+8 \mathrm{i}\left(\nabla^{\alpha} \mathcal{S}\right) \nabla_{\alpha} \sigma+8 \mathrm{i} \mathcal{S} \nabla^{2} \sigma .
\end{aligned}
$$

After some involved algebra, we end up with the super-Weyl variation of $\boldsymbol{\Sigma}_{\mathrm{T}}$ :

$$
\begin{aligned}
\delta_{\sigma} \Sigma_{\mathrm{T}}=\frac{1}{2} E^{\gamma} E^{b} E^{a} \varepsilon_{a b c}\{ & -4(1+\lambda)^{2}(1-3 \lambda)\left(\gamma^{c}\right)_{\gamma}{ }^{\delta} \mathcal{S}^{2} \nabla_{\delta} \sigma+(1+\lambda) \mathrm{i}\left(\gamma^{c}\right)^{\delta \rho} C_{\gamma \delta \rho} \nabla^{2} \sigma \\
+ & \frac{2 \mathrm{i}(1+\lambda)(1-3 \lambda)}{3}\left(\gamma^{c}\right)_{\gamma}{ }^{\delta}\left(\nabla_{\delta} \mathcal{S}\right) \nabla^{2} \sigma-4(1+\lambda)(1-\lambda) \mathcal{S} \nabla^{c} \nabla_{\gamma} \sigma \\
& \left.-4 \lambda(1+\lambda) \varepsilon^{c d e}\left(\gamma_{d}\right)_{\gamma}{ }^{\delta} \mathcal{S} \nabla_{e} \nabla_{\delta} \sigma\right\} \\
+\frac{1}{6} E^{c} E^{b} E^{a} \varepsilon_{a b c}\{ & -2 \mathrm{i}\left(\gamma^{d}\right)_{\alpha \beta} C^{\alpha \beta \gamma} \nabla_{d} \nabla_{\gamma} \sigma-\frac{4 \mathrm{i}\left(1-6 \lambda-3 \lambda^{2}\right)}{3}\left(\gamma^{d}\right)^{\gamma \delta}\left(\nabla_{\gamma} \mathcal{S}\right) \nabla_{d} \nabla_{\delta} \sigma \\
& +(1+\lambda)(1-\lambda)\left(-12 \lambda \mathrm{i} \mathcal{S}^{2} \nabla^{2} \sigma+\left(\nabla^{2} \mathcal{S}\right) \nabla^{2} \sigma-4 \mathcal{S} \nabla^{a} \nabla_{a} \sigma\right) \\
& \left.+12(1+\lambda)\left(1-2 \lambda-\lambda^{2}\right) \mathrm{i} \mathcal{S}\left(\nabla^{\alpha} \mathcal{S}\right) \nabla_{\alpha} \sigma\right\} .
\end{aligned}
$$

This variation is non-zero for any value of $\lambda$.

\subsection{Chern-Simons three-form}

We have just constructed the torsion-induced three-form $\Sigma_{\mathrm{T}}$ which solves the equation 4.13). The same equation has another natural solution given by a Lorentz Chern-Simons three-form defined by

$$
\boldsymbol{\Sigma}_{\mathrm{CS}}:=\boldsymbol{\Omega}^{c} \boldsymbol{R}_{c}+\frac{1}{6} \varepsilon_{a b c} \boldsymbol{\Omega}^{a} \boldsymbol{\Omega}^{b} \boldsymbol{\Omega}^{c}=\frac{2}{5}\left[\boldsymbol{\Omega}_{A}{ }^{B} \boldsymbol{R}_{B}{ }^{A}+\frac{1}{3} \boldsymbol{\Omega}_{A}{ }^{B} \boldsymbol{\Omega}_{B}{ }^{C} \boldsymbol{\Omega}_{C}{ }^{A}\right] .
$$

Indeed, using the structure equation

$$
\mathrm{d} \boldsymbol{R}_{A}{ }^{B}=\boldsymbol{R}_{A}{ }^{C} \boldsymbol{\Omega}_{C}{ }^{B}-\boldsymbol{\Omega}_{A}{ }^{C} \boldsymbol{R}_{C}{ }^{B},
$$


it is easy to check that

$$
\mathrm{d} \boldsymbol{\Sigma}_{\mathrm{CS}}=\boldsymbol{R}^{2}
$$

There are two main differences between the three-forms $\boldsymbol{\Sigma}_{\mathrm{T}}$ and $\boldsymbol{\Sigma}_{\mathrm{C} S}$. First of all, the components of $\boldsymbol{\Sigma}_{\mathrm{T}}$ are tensors constructed in terms of the torsion and its covariant derivatives, while the components of the Chern-Simons three-form $\boldsymbol{\Sigma}_{\mathrm{CS}}$ involve the naked connection. Secondly, the three-form $\boldsymbol{\Sigma}_{\mathrm{T}}$ is invariant under the local Lorentz transformations, while $\Sigma_{\mathrm{CS}}$ changes by an exact term.

The explicit form of $\boldsymbol{\Sigma}_{\mathrm{CS}}$ is as follows:

$$
\begin{aligned}
& \boldsymbol{\Sigma}_{\mathrm{CS}}=\frac{1}{6} E^{\gamma} E^{\beta} E^{\alpha}\left\{12 \mathrm{i}(1+\lambda)\left(\gamma^{a}\right)_{\alpha \beta} \mathcal{S} \boldsymbol{\Omega}_{\gamma a}-\varepsilon_{a b c} \boldsymbol{\Omega}_{\alpha}{ }^{a} \boldsymbol{\Omega}_{\beta}{ }^{b} \boldsymbol{\Omega}_{\gamma}{ }^{c}\right\} \\
& +\frac{1}{2} E^{\gamma} E^{\beta} E^{a}\left\{\left[2\left(\gamma_{a}\right)_{\beta}{ }^{\alpha}\left(\gamma^{b}\right)^{\delta \rho} C_{\alpha \delta \rho}+\frac{4}{3}\left((1+3 \lambda)\left(\nabla_{\beta} \mathcal{S}\right) \delta_{a}^{c}+2 \varepsilon_{a b}{ }^{c}\left(\gamma^{b}\right)_{\beta}{ }^{\alpha}\left(\nabla_{\alpha} \mathcal{S}\right)\right)\right] \boldsymbol{\Omega}_{\gamma c}\right. \\
& \left.+4 \mathrm{i}(1+\lambda)\left(\gamma^{c}\right)_{\beta \gamma} \mathcal{S} \boldsymbol{\Omega}_{a c}-\varepsilon_{b c d} \boldsymbol{\Omega}_{a}{ }^{b} \boldsymbol{\Omega}_{\beta}{ }^{c} \boldsymbol{\Omega}_{\gamma}{ }^{d}\right\} \\
& +\frac{1}{2} E^{\gamma} E^{b} E^{a} \varepsilon_{a b c}\left\{\left[-\frac{\mathrm{i}}{2}\left(\gamma^{c}\right)^{\alpha \beta}\left(\gamma^{d}\right)^{\delta \rho} \nabla_{(\alpha} C_{\beta \delta \rho)}-\eta^{c d}\left(\frac{2 \mathrm{i}}{3}\left(\nabla^{2} \mathcal{S}\right)+4\left(1-\lambda^{2}\right) \mathcal{S}^{2}\right)\right.\right. \\
& \left.+2 \lambda \varepsilon^{c e d}\left(\nabla_{e} \mathcal{S}\right)\right] \Omega_{\gamma d} \\
& +\left[\varepsilon^{c d f}\left(\gamma_{f}\right)_{\gamma \alpha}\left(\gamma^{e}\right)_{\delta \rho} C^{\alpha \delta \rho}-\frac{2(1+3 \lambda)}{3}\left(\nabla_{\gamma} \mathcal{S}\right) \varepsilon^{c d e}\right. \\
& \left.-4 \eta^{e[c}\left(\gamma^{d]}\right)_{\gamma}{ }^{\alpha}\left(\nabla_{\alpha} \mathcal{S}\right)\right] \boldsymbol{\Omega}_{d e} \\
& \left.+\frac{1}{2} \varepsilon^{c d_{1} d_{2}} \varepsilon_{e_{1} e_{2} e_{3}} \Omega_{d_{1}}^{e_{1}} \Omega_{d_{2}}{ }^{e_{2}} \Omega_{\gamma}{ }^{e_{3}}\right\} \\
& +\frac{1}{6} E^{c} E^{b} E^{a} \varepsilon_{a b c}\left\{\left[-\frac{\mathrm{i}}{2}\left(\gamma^{d}\right)^{\alpha \beta}\left(\gamma^{e}\right)^{\gamma \delta} \nabla_{(\alpha} C_{\beta \gamma \delta)}-\eta^{d e}\left(\frac{2 \mathrm{i}}{3}\left(\nabla^{2} \mathcal{S}\right)+4\left(1-\lambda^{2}\right) \mathcal{S}^{2}\right)\right.\right. \\
& \left.-2 \lambda \varepsilon^{\operatorname{def}}\left(\nabla_{f} \mathcal{S}\right)\right] \boldsymbol{\Omega}_{d e} \\
& \left.+\frac{1}{6} \varepsilon_{a b c} \varepsilon^{d_{1} d_{2} d_{3}} \varepsilon_{e_{1} e_{2} e_{3}} \Omega_{d_{1}}^{e_{1}} \Omega_{d_{2}}^{e_{2}} \Omega_{d_{3}}^{e_{3}}\right\} \text {. }
\end{aligned}
$$

We conclude this subsection by giving the expression for the super-Weyl transformation of $\boldsymbol{\Sigma}_{\mathrm{CS}}$. In computing its variation, we can ignore all contributions that are exact three-forms. This considerably simplifies the calculation if we make use of the identity

$$
\delta_{\sigma} \boldsymbol{\Sigma}_{\mathrm{CS}}=2\left(\delta_{\sigma} \boldsymbol{\Omega}^{a}\right) \boldsymbol{R}_{a}+\text { exact three-form }
$$

The result of the calculation is

$$
\delta_{\sigma} \Sigma_{\mathrm{CS}}=\frac{1}{2} E^{\gamma} E^{\beta} E^{a}\left\{-4 \varepsilon_{a b c}\left(\gamma^{b}\right)_{\beta \gamma}\left(\gamma^{c}\right)_{\rho \alpha} C^{\rho \alpha \delta} \nabla_{\delta} \sigma+\frac{4(1+3 \lambda)}{3} \varepsilon_{a b c}\left(\gamma^{b}\right)_{\beta \gamma}\left(\gamma^{c}\right)^{\rho \alpha}\left(\nabla_{\alpha} \mathcal{S}\right) \nabla_{\rho} \sigma\right.
$$




$$
\begin{aligned}
& -4(1-\lambda)\left(\gamma_{a}\right)_{\beta \gamma}\left(\nabla^{\alpha} \mathcal{S}\right) \nabla_{\alpha} \sigma-4 \lambda(1+\lambda)\left(\gamma_{a}\right)_{\beta \gamma} \mathcal{S} \nabla^{2} \sigma \\
& \left.+8 \mathrm{i}(1+\lambda) \varepsilon_{a b c}\left(\gamma^{b}\right)_{\beta \gamma} \mathcal{S} \nabla^{c} \sigma\right\} \\
& +\frac{1}{2} E^{\gamma} E^{b} E^{a} \varepsilon_{a b c}\left\{-2 \mathrm{i}\left(\gamma^{c}\right)^{\alpha \beta}\left(\nabla_{(\alpha} C_{\beta \gamma \delta)}\right) \nabla^{\delta} \sigma+\lambda \mathrm{i}\left(\gamma^{c}\right)^{\delta \rho} C_{\gamma \delta \rho} \nabla^{2} \sigma\right. \\
& +2\left(\gamma_{d}\right)_{\gamma \alpha}\left(\gamma^{c}\right)_{\delta \rho} C^{\alpha \delta \rho} \nabla^{d} \sigma-\left(\gamma^{c}\right)_{\gamma}{ }^{\rho}\left[\frac{4 \mathrm{i}}{3}\left(\nabla^{2} \mathcal{S}\right)+8\left(1-\lambda^{2}\right) \mathcal{S}^{2}\right] \nabla_{\rho} \sigma \\
& -4 \lambda \varepsilon^{c d e}\left(\gamma_{d}\right)_{\gamma}{ }^{\rho}\left(\nabla_{e} \mathcal{S}\right) \nabla_{\rho} \sigma-\frac{8 \lambda \mathrm{i}}{3}\left(\gamma^{c}\right)_{\gamma \alpha}\left(\nabla^{\alpha} \mathcal{S}\right) \nabla^{2} \sigma \\
& \left.+\frac{8}{3}(1+3 \lambda)\left(\nabla_{\gamma} \mathcal{S}\right) \nabla^{c} \sigma-\frac{8}{3} \varepsilon^{c d e}\left(\gamma_{d}\right)_{\gamma \alpha}\left(\nabla^{\alpha} \mathcal{S}\right) \nabla_{e} \sigma\right\} \\
& +\frac{1}{6} E^{c} E^{b} E^{a} \varepsilon_{a b c}\left\{8 \lambda\left(\nabla_{e} \mathcal{S}\right) \nabla^{e} \sigma+2 \lambda\left(\nabla^{2} \mathcal{S}\right) \nabla^{2} \sigma-12 \mathrm{i} \lambda(1+\lambda)(1-\lambda) \mathcal{S}^{2} \nabla^{2} \sigma\right\} \\
& \text { +exact three-form . }
\end{aligned}
$$

\subsection{Closed three-forms}

An immediate corollary of the results obtained so far is that the three-form defined by

$$
\Sigma:=\Sigma_{\mathrm{T}}-\Sigma_{\mathrm{CS}}
$$

is closed,

$$
\mathrm{d} \boldsymbol{\Sigma}=0
$$

Now one can see the advantage of using the deformed covariant derivatives $\nabla_{A}$, eq. 4.2a, which depend on the parameter $\lambda$. The crucial observation is that the three-form $\Sigma$ is closed for any value of $\lambda$, and so are its partial derivatives with respect to $\lambda$. Because $\Sigma$ is polynomial in $\lambda$, by differentiating $\Sigma$ with respect to $\lambda$ we generate a finite number of new closed three-forms. In particular, the explicit form of $\Sigma$ is

$$
\begin{aligned}
& \boldsymbol{\Sigma}=\frac{1}{6} E^{\gamma} E^{\beta} E^{\alpha}\{\left.-12 \mathrm{i}(1+\lambda)\left(\gamma^{a}\right)_{\alpha \beta} \mathcal{S} \boldsymbol{\Omega}_{\gamma a}+\varepsilon_{a b c} \boldsymbol{\Omega}_{\alpha}{ }^{a} \boldsymbol{\Omega}_{\beta}{ }^{b} \boldsymbol{\Omega}_{\gamma}{ }^{c}\right\} \\
&+\frac{1}{2} E^{\gamma} E^{\beta} E^{a}\left\{\left[\frac{8}{3} \varepsilon_{a b}{ }^{c}\left(\gamma^{b}\right)_{\beta \alpha}\left(\nabla^{\alpha} \mathcal{S}\right)-\frac{4(1+3 \lambda)}{3}\left(\nabla_{\beta} \mathcal{S}\right) \delta_{a}^{c}-2\left(\gamma_{a}\right)_{\beta}{ }^{\alpha}\left(\gamma^{c}\right)^{\delta \rho} C_{\alpha \delta \rho}\right] \boldsymbol{\Omega}_{\gamma c}\right. \\
&-\left.4 \mathrm{i}(1+\lambda)\left(\gamma^{c}\right)_{\beta \gamma} \mathcal{S} \boldsymbol{\Omega}_{a c}+\varepsilon_{b c d} \boldsymbol{\Omega}_{a}{ }^{b} \boldsymbol{\Omega}_{\beta}{ }^{c} \boldsymbol{\Omega}_{\gamma}{ }^{d}\right\} \\
&+\frac{1}{2} E^{\gamma} E^{b} E^{a} \varepsilon_{a b c}\{-4(1+\lambda)\left(\gamma^{c}\right)^{\delta \rho} C_{\gamma \delta \rho} \mathcal{S}-\frac{8(1+\lambda)(1-3 \lambda)}{3}\left(\gamma^{c}\right)_{\gamma}{ }^{\delta}\left(\nabla_{\delta} \mathcal{S}\right) \mathcal{S} \\
&+ {\left[\frac{\mathrm{i}}{2}\left(\gamma^{c}\right)^{\alpha \beta}\left(\gamma^{e}\right)^{\rho \delta} \nabla_{(\alpha} C_{\beta \rho \delta)}+\left(\frac{2 \mathrm{i}}{3}\left(\nabla^{2} \mathcal{S}\right)+4\left(1-\lambda^{2}\right) \mathcal{S}^{2}\right) \eta^{c e}\right.} \\
&\left.-2 \lambda \varepsilon^{c d e}\left(\nabla_{d} \mathcal{S}\right)\right] \boldsymbol{\Omega}_{\gamma e}
\end{aligned}
$$




$$
\begin{aligned}
+ & {\left[\varepsilon^{c d e}\left(\gamma_{e}\right)_{\gamma}{ }^{\alpha}\left(\gamma^{f}\right)^{\delta \rho} C_{\alpha \delta \rho}+\frac{2(1+3 \lambda)}{3}\left(\nabla_{\gamma} \mathcal{S}\right) \varepsilon^{c d f}\right.} \\
& \left.+\frac{8}{3} \eta^{f[c}\left(\gamma^{d]}\right)_{\gamma}{ }^{\alpha}\left(\nabla_{\alpha} \mathcal{S}\right)\right] \Omega_{d f} \\
& \left.-\frac{1}{2} \varepsilon^{c d e} \varepsilon_{f g h} \Omega_{d}{ }^{f} \Omega_{e}{ }^{g} \Omega_{\gamma}{ }^{h}\right\} \\
+\frac{1}{6} E^{c} E^{b} E^{a} \varepsilon_{a b c}\{ & 2 \mathrm{i} C^{\mu \rho \tau} C_{\mu \rho \tau}+\frac{4 \mathrm{i}\left(1-6 \lambda-3 \lambda^{2}\right)}{3}\left(\nabla^{\gamma} \mathcal{S}\right)\left(\nabla_{\gamma} \mathcal{S}\right) \\
+ & 4 \mathrm{i}(1+\lambda)(1-\lambda)\left(\nabla^{2} \mathcal{S}\right) \mathcal{S}+16(1+\lambda)\left(1-\lambda^{2}\right) \mathcal{S}^{3} \\
+ & {\left[\frac{\mathrm{i}}{2}\left(\gamma^{d}\right)^{\alpha \beta}\left(\gamma^{f}\right)^{\gamma \delta} \nabla_{(\alpha} C_{\beta \gamma \delta)}+\left(\frac{2 \mathrm{i}}{3}\left(\nabla^{2} \mathcal{S}\right)+4\left(1-\lambda^{2}\right) \mathcal{S}^{2}\right) \eta^{d f}\right.} \\
& \left.-2 \lambda \varepsilon^{d e f}\left(\nabla_{e} \mathcal{S}\right)\right] \Omega_{d f} \\
& \left.-\frac{1}{6} \varepsilon^{e_{1} e_{2} e_{3}} \varepsilon_{d_{1} d_{2} d_{3}} \Omega_{e_{1}}^{d_{1}} \Omega_{e_{2}}{ }^{d_{2}} \boldsymbol{\Omega}_{e_{3}}{ }^{d_{3}}\right\}
\end{aligned}
$$

In computing partial derivatives of $\Sigma$ with respect to $\lambda$, one has to use the identities

$$
\partial_{\lambda} \Omega_{a}^{c}=-2 \delta_{a}^{c} \mathcal{S}, \quad \partial_{\lambda} \nabla_{a}=2 \mathcal{S} \mathcal{M}_{a}
$$

Direct calculations give

$$
\begin{aligned}
\frac{\partial \boldsymbol{\Sigma}}{\partial \lambda}= & \frac{1}{6} E^{\gamma} E^{\beta} E^{\alpha}\left\{-12 \mathrm{i}\left(\gamma^{a}\right)_{\alpha \beta} \mathcal{S} \boldsymbol{\Omega}_{\gamma a}\right\} \\
+ & \frac{1}{2} E^{\gamma} E^{\beta} E^{a}\left\{8 \mathrm{i}(1+\lambda)\left(\gamma_{a}\right)_{\beta \gamma} \mathcal{S}^{2}-4\left(\nabla_{\beta} \mathcal{S}\right) \boldsymbol{\Omega}_{\gamma a}-4 \mathrm{i}\left(\gamma^{c}\right)_{\beta \gamma} \mathcal{S} \boldsymbol{\Omega}_{a c}-2 \varepsilon_{a c d} \mathcal{S} \boldsymbol{\Omega}_{\beta}{ }^{c} \boldsymbol{\Omega}_{\gamma}{ }^{d}\right\} \\
+ & \frac{1}{2} E^{\gamma} E^{b} E^{a} \varepsilon_{a b c}\left\{-2\left(\gamma^{c}\right)^{\delta \rho} C_{\gamma \delta \rho} \mathcal{S}+\frac{16(2+3 \lambda)}{3}\left(\gamma^{c}\right)_{\gamma}{ }^{\delta}\left(\nabla_{\delta} \mathcal{S}\right) \mathcal{S}+2 \varepsilon^{c d f}\left(\nabla_{\gamma} \mathcal{S}\right) \boldsymbol{\Omega}_{d f}\right. \\
& \left.+\left[-8 \lambda \mathcal{S}^{2} \eta^{c e}-2 \varepsilon^{c d e}\left(\nabla_{d} \mathcal{S}\right)\right] \boldsymbol{\Omega}_{\gamma e}+4 \mathcal{S} \boldsymbol{\Omega}_{e}{ }^{[c} \boldsymbol{\Omega}_{\gamma}{ }^{e]}\right\} \\
+\frac{1}{6} E^{c} E^{b} E^{a} \varepsilon_{a b c}\{ & -8 \mathrm{i}(1+\lambda)\left(\nabla^{\gamma} \mathcal{S}\right)\left(\nabla_{\gamma} \mathcal{S}\right)-4 \mathrm{i}(1+2 \lambda)\left(\nabla^{2} \mathcal{S}\right) \mathcal{S} \\
& -8(1+\lambda)(1+3 \lambda) \mathcal{S}^{3}+\left[-8 \lambda \mathcal{S}^{2} \eta^{d f}-2 \varepsilon^{d e f}\left(\nabla_{e} \mathcal{S}\right)\right] \boldsymbol{\Omega}_{d f} \\
& \left.-2 \mathcal{S} \boldsymbol{\Omega}_{d}{ }^{[d} \boldsymbol{\Omega}_{e}{ }^{e]}\right\},
\end{aligned}
$$

as well as

$$
\begin{aligned}
\frac{\partial^{2} \Sigma}{\partial \lambda^{2}}= & \frac{1}{2} E^{\gamma} E^{\beta} E^{a}\left\{\mathrm{i}\left(\gamma_{a}\right)_{\beta \gamma}\left(16 \mathcal{S}^{2}\right)\right\}+\frac{1}{2} E^{\gamma} E^{b} E^{a} \varepsilon_{a b c}\left\{\frac{1}{2}\left(\gamma^{c}\right)_{\gamma}{ }^{\delta} \nabla_{\delta}\left(16 \mathcal{S}^{2}\right)\right\} \\
& +\frac{1}{6} E^{c} E^{b} E^{a} \varepsilon_{a b c}\left\{-\frac{1}{4}\left(\mathrm{i} \nabla^{2}+8 \mathcal{S}\right)\left(16 \mathcal{S}^{2}\right)\right\} \\
= & \Xi\left(16 \mathcal{S}^{2}\right),
\end{aligned}
$$

with the closed three-form $\Xi(\mathcal{L})$ defined by 2.21 . Since the three-form (4.31) is $\lambda$ independent, we conclude that

$$
\frac{\partial^{3} \Sigma}{\partial \lambda^{3}}=0
$$


Thus we have constructed the closed three-forms 4.28, 4.30 and 4.31.

The super-Weyl variations of $\boldsymbol{\Sigma}$ and its first and second derivatives with respect to $\lambda$ can be computed by representing $\delta_{\sigma} \Sigma=\left(\delta_{\sigma} \Sigma_{\mathrm{T}}-\delta_{\sigma} \Sigma_{\mathrm{CS}}\right)$ and then making use of 4.19) and 4.25). It holds that

$$
\begin{aligned}
& \delta_{\sigma} \Sigma=\frac{1}{2} E^{\gamma} E^{\beta} E^{a}\left\{4 \varepsilon_{a b c}\left(\gamma^{b}\right)^{\beta \gamma}\left(\gamma^{c}\right)_{\rho \alpha} C^{\rho \alpha \delta} \nabla_{\delta} \sigma+4(1-\lambda)\left(\gamma_{a}\right)_{\beta \gamma}\left(\nabla^{\alpha} \mathcal{S}\right) \nabla_{\alpha} \sigma\right. \\
& -\frac{4(1+3 \lambda)}{3} \varepsilon_{a b c}\left(\gamma^{b}\right)_{\beta \gamma}\left(\gamma^{c}\right)^{\rho \alpha}\left(\nabla_{\alpha} \mathcal{S}\right) \nabla_{\rho} \sigma+4\left(\lambda+\lambda^{2}\right)\left(\gamma_{a}\right)_{\beta \gamma} \mathcal{S} \nabla^{2} \sigma \\
& \left.+8 \mathrm{i}(1+\lambda) \varepsilon_{a b c}\left(\gamma^{c}\right)_{\beta \gamma} \mathcal{S} \nabla^{b} \sigma\right\} \\
& +\frac{1}{2} E^{\gamma} E^{b} E^{a} \varepsilon_{a b c}\left\{2 \mathrm{i}\left(\gamma^{c}\right)^{\alpha \beta}\left(\nabla_{(\alpha} C_{\beta \gamma \delta)}\right) \nabla^{\delta} \sigma+\mathrm{i}\left(\gamma^{c}\right)^{\delta \rho} C_{\gamma \delta \rho} \nabla^{2} \sigma\right. \\
& -2\left(\gamma^{d}\right)_{\gamma \alpha}\left(\gamma^{c}\right)_{\delta \rho} C^{\alpha \delta \rho} \nabla_{d} \sigma+4\left(1+\lambda+3 \lambda^{2}+3 \lambda^{3}\right)\left(\gamma^{c}\right)_{\gamma}{ }^{\delta} \mathcal{S}^{2} \nabla_{\delta} \sigma \\
& +4 \lambda \varepsilon^{c d e}\left(\gamma_{d}\right)_{\gamma}{ }^{\rho}\left(\nabla_{e} \mathcal{S}\right) \nabla_{\rho} \sigma+\frac{4 \mathrm{i}}{3}\left(\gamma^{c}\right)_{\gamma}{ }^{\rho}\left(\nabla^{2} \mathcal{S}\right) \nabla_{\rho} \sigma \\
& +\frac{2 \mathrm{i}\left(1-6 \lambda-3 \lambda^{2}\right)}{3}\left(\gamma^{c}\right)_{\gamma}{ }^{\delta}\left(\nabla_{\delta} \mathcal{S}\right) \nabla^{2} \sigma-4\left(1-\lambda^{2}\right) \mathcal{S} \nabla^{c} \nabla_{\gamma} \sigma \\
& -4\left(\lambda+\lambda^{2}\right) \varepsilon^{c d e}\left(\gamma_{d}\right)_{\gamma}{ }^{\delta} \mathcal{S} \nabla_{e} \nabla_{\delta} \sigma-\frac{8}{3}(1+3 \lambda)\left(\nabla_{\gamma} \mathcal{S}\right) \nabla^{c} \sigma \\
& \left.+\frac{8}{3} \varepsilon^{c d e}\left(\gamma_{d}\right)_{\gamma \alpha}\left(\nabla^{\alpha} \mathcal{S}\right) \nabla_{e} \sigma\right\} \\
& +\frac{1}{6} E^{c} E^{b} E^{a} \varepsilon_{a b c}\left\{-2 \mathrm{i}\left(\gamma^{d}\right)_{\alpha \rho} C^{\alpha \rho \tau} \nabla_{d} \nabla_{\tau} \sigma+12 \mathrm{i}\left(1-\lambda-3 \lambda^{2}-\lambda^{3}\right) \mathcal{S}\left(\nabla^{\alpha} \mathcal{S}\right) \nabla_{\alpha} \sigma\right. \\
& +\left(1-2 \lambda-\lambda^{2}\right)\left(\nabla^{2} \mathcal{S}\right) \nabla^{2} \sigma-4\left(1-\lambda^{2}\right) \mathcal{S} \nabla^{e} \nabla_{e} \sigma \\
& \left.-8 \lambda\left(\nabla^{e} \mathcal{S}\right) \nabla_{e} \sigma-\frac{4 \mathrm{i}\left(1-6 \lambda-3 \lambda^{2}\right)}{3}\left(\gamma^{d}\right)^{\gamma \delta}\left(\nabla_{\gamma} \mathcal{S}\right) \nabla_{d} \nabla_{\delta} \sigma\right\} \\
& \text { +exact three-form , }
\end{aligned}
$$

and

$$
\begin{aligned}
\delta_{\sigma} \frac{\partial \boldsymbol{\Sigma}}{\partial \lambda}=\frac{1}{2} E^{\gamma} E^{\beta} E^{a}\{ & -4\left(\gamma_{a}\right)_{\beta \gamma}\left(\nabla^{\alpha} \mathcal{S}\right) \nabla_{\alpha} \sigma-4 \varepsilon_{a b c}\left(\gamma^{b}\right)_{\beta \gamma}\left(\gamma^{c}\right)^{\rho \alpha}\left(\nabla_{\alpha} \mathcal{S}\right) \nabla_{\rho} \sigma \\
+ & \left.4(1+2 \lambda)\left(\gamma_{a}\right)_{\beta \gamma} \mathcal{S} \nabla^{2} \sigma+8 \mathrm{i} \varepsilon_{a b c}\left(\gamma^{c}\right)_{\beta \gamma} \mathcal{S} \nabla^{b} \sigma\right\} \\
+\frac{1}{2} E^{\gamma} E^{b} E^{a} \varepsilon_{a b c}\{ & 8\left(1+2 \lambda+3 \lambda^{2}\right)\left(\gamma^{c}\right)_{\gamma}{ }^{\delta} \mathcal{S}^{2} \nabla_{\delta} \sigma+4 \varepsilon^{c d e}\left(\gamma_{d}\right)_{\gamma}{ }^{\delta}\left(\nabla_{e} \mathcal{S}\right) \nabla_{\delta} \sigma \\
& -4(1+\lambda) \mathrm{i}\left(\gamma^{c}\right)_{\gamma}{ }^{\delta}\left(\nabla_{\delta} \mathcal{S}\right) \nabla^{2} \sigma+8 \lambda \mathcal{S} \nabla^{c} \nabla_{\gamma} \sigma-8\left(\nabla_{\gamma} \mathcal{S}\right) \nabla^{c} \sigma \\
& \left.-4(1+2 \lambda) \varepsilon^{c d e}\left(\gamma_{d}\right)_{\gamma}{ }^{\delta} \mathcal{S} \nabla_{e} \nabla_{\delta} \sigma\right\} \\
+\frac{1}{6} E^{c} E^{b} E^{a} \varepsilon_{a b c}\{ & -8 \mathrm{i}\left(2+6 \lambda+3 \lambda^{2}\right) \mathcal{S}\left(\nabla^{\alpha} \mathcal{S}\right) \nabla_{\alpha} \sigma-2(1+\lambda)\left(\nabla^{2} \mathcal{S}\right) \nabla^{2} \sigma \\
& \left.-8\left(\nabla^{e} \mathcal{S}\right) \nabla_{e} \sigma+8 \mathrm{i}(1+\lambda)\left(\gamma^{d}\right)^{\gamma \delta}\left(\nabla_{\gamma} \mathcal{S}\right) \nabla_{d} \nabla_{\delta} \sigma+8 \lambda \mathcal{S} \nabla^{e} \nabla_{e} \sigma\right\}
\end{aligned}
$$


and finally

$$
\begin{aligned}
& \delta_{\sigma} \frac{\partial^{2} \Sigma}{\partial \lambda^{2}}=\frac{1}{2} E^{\gamma} E^{\beta} E^{a}\left\{8\left(\gamma_{a}\right)_{\beta \gamma} \mathcal{S} \nabla^{2} \sigma\right\} \\
& +\frac{1}{2} E^{\gamma} E^{b} E^{a} \varepsilon_{a b c}\left\{8(1+3 \lambda)\left(\gamma^{c}\right)_{\gamma}{ }^{\delta} \mathcal{S}^{2} \nabla_{\delta} \sigma-4 \mathrm{i}\left(\gamma^{c}\right)_{\gamma}{ }^{\delta}\left(\nabla_{\delta} \mathcal{S}\right) \nabla^{2} \sigma\right. \\
& \left.+8 \mathcal{S} \nabla^{c} \nabla_{\gamma} \sigma-8 \varepsilon^{c d e}\left(\gamma_{d}\right)_{\gamma}{ }^{\delta} \mathcal{S} \nabla_{e} \nabla_{\delta} \sigma\right\} \\
& +\frac{1}{6} E^{c} E^{b} E^{a} \varepsilon_{a b c}\left\{-24 \mathrm{i}(1+\lambda) \mathcal{S}\left(\nabla^{\alpha} \mathcal{S}\right) \nabla_{\alpha} \sigma-2\left(\nabla^{2} \mathcal{S}\right) \nabla^{2} \sigma\right. \\
& \left.+8 \mathrm{i}\left(\gamma^{d}\right)^{\gamma \delta}\left(\nabla_{\gamma} \mathcal{S}\right) \nabla_{d} \nabla_{\delta} \sigma+8 \mathcal{S} \nabla^{a} \nabla_{a} \sigma\right\} \\
& \text { +exact three-form . }
\end{aligned}
$$

It is seen that each of the closed three-forms constructed varies non-trivially under the super-Weyl transformations. However, the crucial point is that, for any value of $\lambda$, there exists a linear combination of these three-forms which is super-Weyl invariant modulo an exact three-form. One may check that the three-form

$$
\mathfrak{J}=\boldsymbol{\Sigma}-(1+\lambda) \frac{\partial \boldsymbol{\Sigma}}{\partial \lambda}+\frac{\left(3+2 \lambda+\lambda^{2}\right)}{2} \frac{\partial^{2} \boldsymbol{\Sigma}}{\partial \lambda^{2}}
$$

possesses the following properties:

$$
\begin{aligned}
\mathrm{d} \mathfrak{J} & =0, \\
\delta_{\sigma} \mathfrak{J} & =\text { exact three-form }, \\
\frac{\partial \mathfrak{J}}{\partial \lambda} & =0 .
\end{aligned}
$$

To prove 4.37b we can proceed in two steps. First, we check that the super-Weyl variation of $\mathfrak{J}_{a \beta \gamma}$ can be represented as

$$
\delta_{\sigma} \mathfrak{J}_{a \beta \gamma}=8\left(\gamma_{a}\right)_{\beta \gamma} \nabla^{\alpha}\left(\mathcal{S} \nabla_{\alpha} \sigma\right)+\varepsilon_{a b c}\left(\gamma^{b}\right)_{\beta \gamma} V^{c}
$$

where $V^{c}$ is a vector that can easily be computed using the relations 4.33-4.36). Next, making use of eqs. B.3 - B.4 and ignoring exact terms, we observe that $\delta_{\sigma} \mathfrak{J}$ is an exact three-form.

Let us write down the final expression for $\mathfrak{J}$ $\mathfrak{J}=\frac{1}{6} E^{\gamma} E^{\beta} E^{\alpha}\left\{\varepsilon_{a b c} \boldsymbol{\Omega}_{\alpha}{ }^{a} \boldsymbol{\Omega}_{\beta}{ }^{b} \boldsymbol{\Omega}_{\gamma}{ }^{c}\right\}$ 


$$
\begin{aligned}
&+\frac{1}{2} E^{\gamma} E^{\beta} E^{a}\left\{16 \mathrm{i}\left(\gamma_{a}\right)_{\beta \gamma} \mathcal{S}^{2}+2(1+\lambda) \varepsilon_{a c d} \mathcal{S} \boldsymbol{\Omega}_{\beta}{ }^{c} \boldsymbol{\Omega}_{\gamma}{ }^{d}+\varepsilon_{b c d} \boldsymbol{\Omega}_{a}{ }^{b} \boldsymbol{\Omega}_{\beta}{ }^{c} \boldsymbol{\Omega}_{\gamma}{ }^{d}\right. \\
&+ {\left.\left[\frac{8}{3}\left(\nabla_{\beta} \mathcal{S}\right) \delta_{a}^{c}-\frac{8}{3} \varepsilon_{a b}{ }^{c}\left(\gamma^{b}\right)_{\beta}{ }^{\alpha}\left(\nabla_{\alpha} \mathcal{S}\right)+2\left(\gamma_{a}\right)_{\beta \alpha}\left(\gamma^{c}\right)_{\delta \rho} C^{\alpha \delta \rho}\right] \boldsymbol{\Omega}_{\gamma c}\right\} } \\
&+\frac{1}{2} E^{\gamma} E^{b} E^{a} \varepsilon_{a b c}\left\{-2(1+\lambda)\left(\gamma^{c}\right)^{\gamma \delta} C_{\beta \gamma \delta} \mathcal{S}+\frac{16(2-\lambda)}{3}\left(\gamma^{c}\right)_{\beta}{ }^{\gamma}\left(\nabla_{\gamma} \mathcal{S}\right) \mathcal{S}\right. \\
&+\left[\frac{\mathrm{i}}{2}\left(\gamma^{c}\right)^{\alpha \beta}\left(\gamma^{e}\right)^{\delta \rho} \nabla_{(\alpha} C_{\beta \delta \rho)}+\left(\frac{2 \mathrm{i}}{3}\left(\nabla^{2} \mathcal{S}\right)+4(1+\lambda)^{2} \mathcal{S}^{2}\right) \eta^{c e}+2 \varepsilon^{c d e}\left(\nabla_{d} \mathcal{S}\right)\right] \boldsymbol{\Omega}_{\gamma e} \\
&+\left[\varepsilon^{c d e}\left(\gamma_{e}\right)_{\gamma}{ }^{\alpha}\left(\gamma^{f}\right)^{\delta \rho} C_{\alpha \delta \rho}-\frac{4}{3}\left(\nabla_{\gamma} \mathcal{S}\right) \varepsilon^{c d f}+\frac{8}{3} \eta^{f[c}\left(\gamma^{d]}\right)_{\gamma}{ }^{\alpha}\left(\nabla_{\alpha} \mathcal{S}\right)\right] \boldsymbol{\Omega}_{d f} \\
&\left.-4(1+\lambda) \mathcal{S} \boldsymbol{\Omega}_{e}{ }^{[c} \boldsymbol{\Omega}_{\gamma}{ }^{e]}-\frac{1}{2} \varepsilon^{c d e} \varepsilon_{f g h} \boldsymbol{\Omega}_{d}{ }^{f} \boldsymbol{\Omega}_{e}{ }^{g} \boldsymbol{\Omega}_{\gamma}{ }^{h}\right\} \\
&+\frac{1}{6} E^{c} E^{b} E^{a} \varepsilon_{a b c}\left\{2 \mathrm{i} C^{\mu \rho \tau} C_{\mu \rho \tau}-\frac{8 \mathrm{i}}{3}\left(\nabla^{\gamma} \mathcal{S}\right)\left(\nabla_{\gamma} \mathcal{S}\right)-4 \mathrm{i}(1-\lambda)\left(\nabla^{2} \mathcal{S}\right) \mathcal{S}\right. \\
&-8\left(3-3 \lambda-3 \lambda^{2}-\lambda^{3}\right) \mathcal{S}^{3} \\
&+\left[\frac{\mathrm{i}}{2}\left(\gamma^{d}\right)^{\alpha \beta}\left(\gamma^{f}\right)^{\gamma \delta} \nabla_{(\alpha} C_{\beta \gamma \delta)}+\left(\frac{2 \mathrm{i}}{3}\left(\nabla^{2} \mathcal{S}\right)+4(1+\lambda)^{2} \mathcal{S}^{2}\right) \eta^{d f}+2 \varepsilon^{d e f}\left(\nabla_{e} \mathcal{S}\right)\right] \boldsymbol{\Omega}_{d f} \\
&\left.+2(1+\lambda) \mathcal{S} \Omega_{d}{ }^{[d} \boldsymbol{\Omega}_{e}{ }^{e]}-\frac{1}{6} \varepsilon^{e_{1} e_{2} e_{3}} \varepsilon_{d_{1} d_{2} d_{3}} \boldsymbol{\Omega}_{e_{1}}{ }^{d_{1}} \boldsymbol{\Omega}_{e_{2}}{ }^{d_{2}} \boldsymbol{\Omega}_{e_{3}}{ }^{d_{3}}\right\} .
\end{aligned}
$$

Since the three-form $\mathfrak{J}$ is $\lambda$-independent, any convenient value of $\lambda$ may be used in order to compute $\mathfrak{J}$. Setting $\lambda=0$ gives

$$
\begin{aligned}
& \mathfrak{J}=\frac{1}{6} E^{\gamma} E^{\beta} E^{\alpha}\{\left.\varepsilon_{a b c} \Omega_{\alpha}{ }^{a} \Omega_{\beta}{ }^{b} \Omega_{\gamma}{ }^{c}\right\} \\
&+\frac{1}{2} E^{\gamma} E^{\beta} E^{a}\{ 16 \mathrm{i}\left(\gamma_{a}\right)_{\beta \gamma} \mathcal{S}^{2}+2 \varepsilon_{a c d} \mathcal{S} \Omega_{\beta}{ }^{c} \Omega_{\gamma}{ }^{d}+\varepsilon_{b c d} \Omega_{a}{ }^{b} \Omega_{\beta}{ }^{c} \Omega_{\gamma}{ }^{d} \\
&\left.+\left[\frac{8}{3} \varepsilon_{a b}{ }^{c}\left(\gamma^{b}\right)_{\beta \alpha}\left(\mathcal{D}^{\alpha} \mathcal{S}\right)+\frac{8}{3}\left(\mathcal{D}_{\beta} \mathcal{S}\right) \delta_{a}^{c}-2\left(\gamma_{a}\right)_{\beta}{ }^{\alpha}\left(\gamma^{c}\right)^{\delta \rho} C_{\alpha \delta \rho}\right] \Omega_{\gamma c}\right\} \\
&+\frac{1}{2} E^{\gamma} E^{b} E^{a} \varepsilon_{a b c}\left\{-2\left(\gamma^{c}\right)^{\delta \rho} C_{\gamma \delta \rho} \mathcal{S}+\frac{32}{3}\left(\gamma^{c}\right)_{\gamma}{ }^{\delta}\left(\mathcal{D}_{\delta} \mathcal{S}\right) \mathcal{S}\right. \\
&+ {\left[\frac{\mathrm{i}}{2}\left(\gamma^{c}\right)^{\alpha \beta}\left(\gamma^{e}\right)^{\delta \rho} \mathcal{D}_{(\alpha} C_{\beta \delta \rho}+\left(\frac{2 \mathrm{i}}{3}\left(\mathcal{D}^{2} \mathcal{S}\right)+4 \mathcal{S}^{2}\right) \eta^{c e}+2 \varepsilon^{c d e}\left(\mathcal{D}_{d} \mathcal{S}\right)\right] \Omega_{\gamma e} } \\
&+ {\left[\varepsilon^{c d e}\left(\gamma_{e}\right)_{\gamma}{ }^{\alpha}\left(\gamma^{f}\right)^{\delta \rho} C_{\alpha \delta \rho}-\frac{4}{3}\left(\mathcal{D}_{\gamma} \mathcal{S}\right) \varepsilon^{c d f}+\frac{8}{3} \eta^{f[c}\left(\gamma^{d]}\right){ }_{\gamma}{ }^{\alpha}\left(\mathcal{D}_{\alpha} \mathcal{S}\right)\right] \Omega_{d f} } \\
&\left.-4 \mathcal{S} \Omega_{e}{ }^{[c} \Omega_{\gamma}{ }^{e]}-\frac{1}{2} \varepsilon^{c d e} \varepsilon_{f g h} \Omega_{d}{ }^{f} \Omega_{e}{ }^{g} \Omega_{\gamma}{ }^{h}\right\} \\
&+\frac{1}{6} E^{c} E^{b} E^{a} \varepsilon_{a b c}\left\{2 \mathrm{i} C^{\alpha \rho \tau} C_{\alpha \rho \tau}-\frac{8 \mathrm{i}}{3}\left(\mathcal{D}^{\gamma} \mathcal{S}\right)\left(\mathcal{D}_{\gamma} \mathcal{S}\right)-4 \mathrm{i}\left(\mathcal{D}^{2} \mathcal{S}\right) \mathcal{S}-24 \mathcal{S}^{3}\right. \\
&+ {\left[\frac{\mathrm{i}}{2}\left(\gamma^{d}\right)^{\alpha \beta}\left(\gamma^{f}\right)^{\gamma \delta} \mathcal{D}_{(\alpha} C_{\beta \gamma \delta)}+\left(\frac{2 \mathrm{i}}{3}\left(\mathcal{D}^{2} \mathcal{S}\right)+4 \mathcal{S}^{2}\right) \eta^{d f}+2 \varepsilon^{d e f}\left(\mathcal{D}_{e} \mathcal{S}\right)\right] \Omega_{d f} } \\
&\left.+2 \mathcal{S} \Omega_{d}{ }^{[d} \Omega_{e}{ }^{e]}-\frac{1}{6} \varepsilon^{e_{1} e_{2} e_{3}} \varepsilon_{d_{1} d_{2} d_{3}} \Omega_{e_{1}}{ }^{d_{1}} \Omega_{e_{2}}{ }^{d_{2}} \Omega_{e_{3}}{ }^{d_{3}}\right\}
\end{aligned}
$$




\subsection{The action principle for conformal supergravity}

The three-form $\mathfrak{J}$ is our main result. Associated with $\mathfrak{J}$ is the action for $\mathcal{N}=1$ conformal supergravity defined via the integration of $\mathfrak{J}$ over spacetime $\mathcal{M}_{3}$ :

$$
S_{\mathrm{CSG}}=\int_{\mathcal{M}_{3}} \mathfrak{J}=\int \mathrm{d}^{3} x\left({ }^{*} \mathfrak{J}\right), \quad{ }^{*} \mathfrak{J}=\frac{1}{3 !} \varepsilon^{m n p} \mathfrak{J}_{m n p}
$$

The action is automatically invariant under the local Lorentz and super-Weyl transformations, since the corresponding variations of the three-form $\mathfrak{J}$ have been shown to be exact. It remains to show that $S_{\mathrm{CSG}}$ is invariant under general coordinate transformations of the curved superspace generated by the vector field $\xi^{C} E_{C}$ in (2.4). It suffices to repeat the four-dimensional proof due to Hasler [12] (see also [14])

$$
\delta_{\xi} \mathfrak{J}=\mathcal{L}_{\xi} \mathfrak{J}=\imath_{\xi} \mathrm{d} \mathfrak{J}+\mathrm{d} \imath_{\xi} \mathfrak{J}=\mathrm{d} \imath_{\xi} \mathfrak{J}
$$

where $\imath_{\xi}$ denotes the interior product and $\mathcal{L}_{\xi}$ the Lie derivative. Since the variation $\delta_{\xi} \mathfrak{J}$ is an exact three-form, the action $S_{\mathrm{CSG}}$ is indeed invariant under superdiffeomorphisms provided $\mathcal{M}_{3}$ has no boundary.

\section{Component action}

In this section we reduce the action (4.41) to the component fields, choosing a special Wess-Zumino gauge, and demonstrate that it coincides with the well-known action for $\mathcal{N}=1$ conformal supergravity [3]. To start with, we elaborate on the component reduction.

\subsection{Components reduction}

Given a superfield $U(z)$ we define its bar-projection $U \mid$ to be the $\theta$-independent term in the expansion of $U(x, \theta)$ in powers of $\theta$ 's,

$$
U|:=U(x, \theta)|_{\theta=0} .
$$

In a similar way we define the bar-projection of the covariant derivatives:

$$
\mathcal{D}_{A}\left|:=E_{A}{ }^{M}\right| \partial_{M}+\frac{1}{2} \Omega_{A}{ }^{b c} \mid \mathcal{M}_{b c} .
$$


The supergravity gauge freedom may be used to algebraically gauge away a number of component fields contained in $\mathcal{D}_{A}$ except those which constitute the Weyl multiplet of conformal supergravity. The supergravity gauge group is spanned by the general coordinate, local Lorentz and super-Weyl transformations.

The freedom to perform general coordinate and local $\mathrm{SL}(2, \mathbb{R})$ transformations can be used to choose a Wess-Zumino gauge of the form

$$
\begin{aligned}
& \mathcal{D}_{\alpha}\left|=\delta_{\alpha}{ }^{\mu} \frac{\partial}{\partial \theta^{\mu}} \Longleftrightarrow \quad E_{\alpha}{ }^{\mu}\right|=\delta_{\alpha}{ }^{\mu}, \quad \Omega_{\alpha}{ }^{b c} \mid=0, \\
& \mathcal{D}_{a}\left|=\mathbf{D}_{a}+\Psi_{a}{ }^{\gamma}(x) \mathcal{D}_{\gamma}\right|
\end{aligned}
$$

where $\mathbf{D}_{a}$ denotes a space-time covariant derivative

$$
\mathbf{D}_{a}=e_{a}+\omega_{a}, \quad e_{a}=e_{a}{ }^{m}(x) \partial_{m}, \quad \omega_{a}=\frac{1}{2} \omega_{a}^{b c}(x) \mathcal{M}_{b c} .
$$

Here the component inverse vielbein $e_{a}{ }^{m}(x)$ and the component vielbein $e_{m}{ }^{a}(x)$ are defined as

$$
e_{a}{ }^{m}:=E_{a}{ }^{m}\left|, \quad e_{m}{ }^{a}:=E_{m}{ }^{a}\right| .
$$

They are related to each other in the standard way

$$
e_{a}{ }^{m} e_{m}{ }^{b}=\delta_{a}^{b}, \quad e_{m}{ }^{a} e_{a}{ }^{n}=\delta_{m}^{n} .
$$

The component Lorentz connection is defined as

$$
\omega_{a}^{b c}:=\Omega_{a}^{b c} \mid .
$$

Finally, the gravitino is defined by the rule:

$$
\Psi_{a}^{\gamma}:=E_{a}^{\gamma}\left|, \quad e_{m}^{a} \Psi_{a}^{\gamma}:=-E_{m}^{\gamma}\right|
$$

The space-time covariant derivatives $\mathbf{D}_{a}$ obey the commutation relations

$$
\left[\mathbf{D}_{a}, \mathbf{D}_{b}\right]=\mathcal{T}_{a b}{ }^{c} \mathbf{D}_{c}+\frac{1}{2} \mathcal{R}_{a b}{ }^{c d} \mathcal{M}_{c d},
$$

with $\mathcal{T}_{a b}{ }^{c}$ the torsion and $\mathcal{R}_{a b}{ }^{c d}$ the curvature. Their explicit expressions are

$$
\begin{aligned}
\mathcal{T}_{a b}{ }^{c} & =\mathcal{C}_{a b}{ }^{c}+2 \omega_{[a b]}{ }^{c}, \\
\mathcal{R}_{a b}{ }^{c d} & =2 e_{[a} \omega_{b]}{ }^{c d}+2 \omega_{[a b]}{ }^{f} \omega_{f}{ }^{c d}+2 \omega_{[a}{ }^{c f} \omega_{b] f}{ }^{d}-\mathcal{T}_{a b}{ }^{f} \omega_{f}{ }^{c d},
\end{aligned}
$$


where $\mathcal{C}_{a b}{ }^{c}$ stands for the anholonomy coefficients,

$$
\left[e_{a}, e_{b}\right]=\mathcal{C}_{a b}{ }^{c} e_{c}, \quad \mathcal{C}_{a b}^{c}=2\left(e_{[a} e_{b]}^{n}\right) e_{n}^{c}
$$

The connection is uniquely determined as a function of the vielbein and torsion, $\omega_{a}^{c d}=$ $\omega_{a}^{c d}(e, \mathcal{T})$. Its explicit form is

$$
\omega_{a b c}=\frac{1}{2}\left[\left(\mathcal{T}_{a b c}-\mathcal{C}_{a b c}\right)-\left(\mathcal{T}_{b c a}-\mathcal{C}_{b c a}\right)+\left(\mathcal{T}_{c a b}-\mathcal{C}_{c a b}\right)\right]
$$

So far we have partially fixed the general coordinate and local Lorentz symmetries. We still have the complete super-Weyl gauge freedom at our disposal. Let us recall the super-Weyl transformation of the torsion superfield $\mathcal{S}$, eq. 2.11a). It follows from (2.11a that we are in a position to choose the gauge

$$
\mathcal{S} \mid=0
$$

The conditions (5.3) and (5.13) constitute the complete Wess-Zumino guage. In this gauge, the only independent component fields are the vielbein and the gravitino, and they comprise the Weyl multiplet of $\mathcal{N}=1$ conformal supergravity. The gauge condition (5.3) does not fix completely the general coordinate and local Lorentz symmetries of the curved superspace. The residual gauge transformations, which preserve the condition (5.3), are spanned by (i) the general coordinate transformations in space-time; (ii) the local Lorentz transformations in space-time; and (iii) the $Q$-supersymmetry transformations. The gauge condition (5.13) only partially fixes the super-Weyl gauge freedom. The residual super-Weyl transformations, which preserve (5.13), are (iv) the space-time Weyl transformations; and (v) the $S$-supersymmetry transformations.

To complete the component reduction, we express the gravitino field strength and the component torsion and curvature in terms of the superfield torsion:

$$
\begin{aligned}
\mathcal{T}_{a b}{ }^{c}= & -2 \mathrm{i}\left(\gamma^{c}\right)_{\gamma \delta} \Psi_{a}{ }^{\gamma} \Psi_{b}{ }^{\delta} \\
\varepsilon_{c}{ }^{a b}\left(\mathbf{D}_{[a} \Psi_{b]}{ }^{\rho}\right)= & {\left[-\frac{\mathrm{i}}{2}\left(\gamma_{c}\right)_{\alpha \beta} C^{\rho \alpha \beta}+\frac{2 \mathrm{i}}{3}\left(\gamma_{c}\right)^{\rho \alpha}\left(\mathcal{D}_{\alpha} \mathcal{S}\right)+\frac{1}{2} \varepsilon_{c}{ }^{a b} \mathcal{T}_{a b}{ }^{d} \Psi_{d}{ }^{\rho}\right] \mid } \\
\varepsilon^{a b c} \mathcal{R}_{b c}{ }^{d}=[ & {\left[\mathrm{i}\left(\gamma^{a}\right)^{\alpha \beta}\left(\gamma^{d}\right)^{\gamma \delta}\left(\mathcal{D}_{(\alpha} C_{\beta \gamma \delta)}\right)+\frac{4 \mathrm{i}}{3}\left(\mathcal{D}^{2} \mathcal{S}\right) \eta^{c d}\right.} \\
& \left.+\varepsilon^{a b c} \Psi_{b}{ }^{\gamma}\left(2\left(\gamma_{c}\right)_{\gamma}{ }^{\delta}\left(\gamma^{d}\right)^{\alpha \beta} C_{\delta \alpha \beta}+\frac{4}{3}\left(\delta_{\gamma}^{\delta} \delta_{c}^{d}+2 \varepsilon_{c e}{ }^{d}\left(\gamma^{e}\right)_{\gamma}{ }^{\delta}\right) \mathcal{D}_{\delta} \mathcal{S}\right)\right] \mid .
\end{aligned}
$$

Here we have denoted $\mathcal{R}_{a b}{ }^{d}=\frac{1}{2} \varepsilon^{d}{ }_{e f} \mathcal{R}_{a b}{ }^{e f}$. Since the torsion is a quadratic polynomial of the gravitino, eq. (5.14a), the Lorentz connection is determined in terms of the vielbein and gravitino, $\omega_{a}^{c d}=\omega_{a}^{c d}(e, \Psi)$. 


\subsection{The action for conformal supergravity in Wess-Zumino gauge}

Our superspace action for $\mathcal{N}=1$ conformal supergravity, eq. (4.41), is constructed in terms of the closed three-form $\mathfrak{J}$ given by 4.40 . We now express it in terms of the component fields. The action can equivalently be rewritten in the following form

$$
S_{\mathrm{CSG}}=\frac{1}{6} \int \mathrm{d}^{3} x e \varepsilon^{a b c}\left[\mathfrak{J}_{a b c}-3 \Psi_{a}^{\gamma} \mathfrak{J}_{b c \gamma}-3 \Psi_{a}{ }^{\beta} \Psi_{b}{ }^{\gamma} \mathfrak{J}_{c \beta \gamma}+\Psi_{a}{ }^{\alpha} \Psi_{b}{ }^{\beta} \Psi_{c}{ }^{\gamma} \mathfrak{J}_{\alpha \beta \gamma}\right] \mid
$$

where $e=\operatorname{det}\left(e_{m}{ }^{a}\right)$ and the gravitino $\Psi_{a}^{\gamma}$ is defined according to (5.8). To compute the integrand, we have to make use of the explicit expressions for the components of the three-form $\mathfrak{J}$, eq. (4.40). In the Wess-Zumino gauge defined by eqs. (5.3a) and (5.13), the result is

$$
\begin{array}{rl}
S_{\mathrm{CSG}}=\int \mathrm{d}^{3} & x e\left\{-2 \mathrm{i} C^{\alpha \beta \gamma} C_{\alpha \beta \gamma}+\frac{8 \mathrm{i}}{3}\left(\mathcal{D}^{\alpha} \mathcal{S}\right) \mathcal{D}_{\alpha} \mathcal{S}+\frac{1}{6} \varepsilon^{a_{1} a_{2} a_{3}} \varepsilon_{b_{1} b_{2} b_{3}} \omega_{a_{1}}{ }^{{ }^{b_{1}}} \omega_{a_{2}}{ }^{b_{2}} \omega_{a_{3}}{ }^{b_{3}}\right. \\
+ & \Psi_{a}{ }^{\alpha}\left[\varepsilon^{a b d}\left(\gamma_{d}\right)_{\alpha}{ }^{\beta}\left(\gamma^{c}\right)^{\gamma \delta} C_{\beta \gamma \delta}+\frac{2}{3}\left(\mathcal{D}_{\alpha} \mathcal{S}\right) \varepsilon^{a b c}+\frac{8}{3} \eta^{c[a}\left(\gamma^{b]}\right)_{\alpha}{ }^{\beta}\left(\mathcal{D}_{\beta} \mathcal{S}\right)\right] \omega_{b c} \\
+ & \left.\left[-\frac{\mathrm{i}}{2}\left(\gamma^{a}\right)^{\alpha \beta}\left(\gamma^{b}\right)^{\gamma \delta} \mathcal{D}_{(\alpha} C_{\beta \gamma \delta)}-\frac{2 \mathrm{i}}{3}\left(\mathcal{D}^{2} \mathcal{S}\right) \eta^{a b}\right] \omega_{a b}\right\} \mid
\end{array}
$$

It only remains to make use of the relations $5.14 \mathrm{~b}$ and $5.14 \mathrm{c}$ to arrive at the final expression for the action:

$$
\begin{aligned}
S_{\mathrm{CSG}}= & \frac{1}{4} \int \mathrm{d}^{3} x e \varepsilon^{a b c}\left\{\omega_{a}{ }^{f g} \mathcal{R}_{b c f g}+\frac{2}{3} \omega_{a f}{ }^{g} \omega_{b g}{ }^{h} \omega_{c h}{ }^{f}\right. \\
& \left.-8 \mathrm{i}\left(\mathbf{D}_{b} \Psi_{c}{ }^{\alpha}-\frac{1}{2} \mathcal{T}_{b c}{ }^{g} \Psi_{g}{ }^{\alpha}\right)\left(\gamma_{d}\right)_{\alpha}{ }^{\beta}\left(\gamma_{a}\right)_{\beta}{ }^{\gamma} \varepsilon^{d e f}\left(\mathbf{D}_{e} \Psi_{f \gamma}-\frac{1}{2} \mathcal{T}_{e f}{ }^{h} \Psi_{h \gamma}\right)\right\}
\end{aligned}
$$

Up to an overall factor of $1 / 4$, this is the well-known action for $\mathcal{N}=1$ conformal supergravity 3 .

\section{Outlook}

In this paper we have presented the new superfield method to construct the action for three-dimensional $\mathcal{N}=1$ conformal supergravity, and thus for $\mathcal{N}=1$ topologically massive supergravity. The power of this method is that it may naturally be generalized to the case of $\mathcal{N}$-extended conformal supergravity. Here we only sketch such a generalization, leaving details for a future publication.

Let $\mathcal{D}_{A}=\left(\mathcal{D}_{a}, \mathcal{D}_{\alpha}^{I}\right)$ be the superspace covariant derivatives, with $I=1, \ldots, \mathcal{N}$, which describe the off-shell $\mathcal{N}$-extended Weyl supermultiplet [9, 10]. Following the conventions 
of [10], we consider a two-parameter deformation of the vector covariant derivative

$$
\mathcal{D}_{\alpha \beta} \rightarrow \nabla_{\alpha \beta}=\mathcal{D}_{\alpha \beta}+\lambda \mathcal{S} \mathcal{M}_{\alpha \beta}+\rho C_{\alpha \beta}{ }^{K L} \mathcal{N}_{K L}
$$

where $\lambda$ and $\rho$ are real parameters, and $\mathcal{S}$ and $C_{\alpha \beta}{ }^{K L}$ are certain dimension-one torsion tensors. The deformed covariant derivatives $\nabla_{A}=\left(\nabla_{a}, \nabla_{\alpha}^{I}\right):=\left(\nabla_{a}, \mathcal{D}_{\alpha}^{I}\right)$ obey the algebra

$$
\left[\nabla_{A}, \nabla_{B}\right\}=\boldsymbol{T}_{A B}{ }^{C} \nabla_{C}+\frac{1}{2} \boldsymbol{R}_{A B}{ }^{c d} \mathcal{M}_{c d}+\frac{1}{2} \boldsymbol{R}_{A B}{ }^{K L} \mathcal{N}_{K L}
$$

with $\boldsymbol{T}_{A B}^{C}$ the torsion, $\boldsymbol{R}_{A B}{ }^{c d}$ the Lorentz curvature and $\boldsymbol{R}_{A B}{ }^{K L}$ the $\mathrm{SO}(\mathcal{N})$ curvature. As a next stage, we have to consider the equation

$$
\mathrm{d} \boldsymbol{\Sigma}=\frac{1}{2} \boldsymbol{R}^{a b} \boldsymbol{R}_{a b}+\frac{\kappa}{2} \boldsymbol{R}^{I J} \boldsymbol{R}_{I J}
$$

with $\kappa$ a real parameter, and look for two solutions $\boldsymbol{\Sigma}_{\mathrm{T}}$ and $\boldsymbol{\Sigma}_{\mathrm{CS}}$. Here $\boldsymbol{\Sigma}_{\mathrm{T}}$ is a three-form constructed in terms of the torsion and curvature tensors and their covariant derivatives, while $\boldsymbol{\Sigma}_{\mathrm{CS}}$ is a standard Chern-Simons three-form. Now, the three-form $\Sigma:=\Sigma_{\mathrm{T}}-\boldsymbol{\Sigma}_{\mathrm{CS}}$ has the following properties (i) $\boldsymbol{\Sigma}$ is closed; and (ii) $\boldsymbol{\Sigma}$ is a polynomial in two variables $\lambda$ and $\rho$. By differentiating $\boldsymbol{\Sigma}$ with respect to $\lambda$ and $\rho$, we will generate a number of closed three-forms. Finally, we have to look for a linear combinations $\mathfrak{J}$ of these closed three-forms, which is super-Weyl invariant modulo exact contributions. The parameter $\kappa$ is expected to be fixed by this requirement. It is also expected that $\mathfrak{J}$ is independent of $\lambda$ and $\rho$, due to its uniqueness. The closed three-form $\mathfrak{J}$ generates the action for $\mathcal{N}$-extended conformal supergravity.

\section{Acknowledgements:}

We thank U. Lindström, M. Roček and I. Sachs for discussions that rekindled our interest in the problem solved in this paper. We are also grateful to Joseph Novak for reading the manuscript. The work of SMK was supported in part by the Australian Research Council under Grant No. DP1096372. The work of GT-M was supported by the Australian Research Council's Discovery Early Career Award (DECRA), project No. DE120101498.

\section{A Supersymmetric action}

For completeness of our presentation, in this appendix we review the structure of the locally supersymmetric action [20] associated with the closed three-form (2.21). This 
action is defined, in complete analogy with 4.41), by integrating the three-form $\Xi$ over the space-time,

$$
S(\mathcal{L})=\int_{\mathcal{M}_{3}} \Xi=\int \mathrm{d}^{3} x\left({ }^{*} \Xi\right), \quad{ }^{*} \Xi=\frac{1}{3 !} \varepsilon^{m n p} \Xi_{m n p}
$$

in accordance with the superform approach to construct supersymmetric invariants [12, [13, 14]. In the Wess-Zumino gauge (5.3a), the action can readily be brought to the form

$$
S(\mathcal{L})=\frac{1}{6} \int \mathrm{d}^{3} x e \varepsilon^{a b c}\left[\Xi_{a b c}-3 \Psi_{a}^{\gamma} \Xi_{b c \gamma}-3 \Psi_{a}{ }^{\beta} \Psi_{b}{ }^{\gamma} \Xi_{c \beta \gamma}+\Psi_{a}{ }^{\alpha} \Psi_{b}{ }^{\beta} \Psi_{c}{ }^{\gamma} \Xi_{\alpha \beta \gamma}\right],
$$

or equivalently

$$
S(\mathcal{L})=\int \mathrm{d}^{3} x e\left[\frac{\mathrm{i}}{4} \mathcal{D}^{2}+2 \mathcal{S}-\frac{1}{2}\left(\gamma^{a}\right)^{\gamma \delta} \Psi_{a \gamma} \mathcal{D}_{\delta}-\frac{\mathrm{i}}{2} \varepsilon^{a b c} \Psi_{a}^{\beta} \Psi_{b}^{\gamma}\left(\gamma_{c}\right)_{\beta \gamma}\right] \mathcal{L}
$$

The locally supersymmetric action A.2a was derived in [20]. Our consideration in section 2 shows that this action is super-Weyl invariant. The component action (A.2b) was first derived in [18.

\section{B Exact three-forms}

Given a two-form $\boldsymbol{F}_{2}=\frac{1}{2} E^{B} E^{A} \boldsymbol{F}_{A B}$, its exterior derivative is

$$
\mathrm{d} \boldsymbol{F}_{2}=\frac{1}{2} E^{C} E^{B} E^{A}\left\{\nabla_{A} \boldsymbol{F}_{B C}-\boldsymbol{T}_{A B}^{D} \boldsymbol{F}_{D C}\right\} .
$$

Making use of the explicit expression for the torsion associated with the covariant derivatives $\nabla_{A}$, we obtain

$$
\begin{aligned}
\mathrm{d} \boldsymbol{F}_{2}= & \frac{1}{6} E^{\gamma} E^{\beta} E^{\alpha}\left\{3 \nabla_{\alpha} \boldsymbol{F}_{\beta \gamma}-6 \mathrm{i}\left(\gamma^{d}\right)_{\alpha \beta} \boldsymbol{F}_{d \gamma}\right\} \\
+ & \frac{1}{2} E^{\gamma} E^{\beta} E^{a}\left\{\nabla_{a} \boldsymbol{F}_{\beta \gamma}-2 \nabla_{\beta} \boldsymbol{F}_{a \gamma}-2(1-\lambda)\left(\gamma_{a}\right)_{(\beta}{ }^{\delta} \boldsymbol{F}_{\gamma) \delta} \mathcal{S}-2 \mathrm{i}\left(\gamma^{c}\right)_{\beta \gamma} \boldsymbol{F}_{c a}\right\} \\
+ & \frac{1}{2} E^{\gamma} E^{b} E^{a} \varepsilon_{a b c}\left\{-\varepsilon^{c d e} \nabla_{d} \boldsymbol{F}_{e \gamma}-\frac{1}{2} \varepsilon^{c d e} \nabla_{\gamma} \boldsymbol{F}_{d e}-4 \lambda \boldsymbol{F}_{\gamma}^{c} \mathcal{S}+(1-\lambda) \varepsilon^{c a b}\left(\gamma_{a}\right)_{\gamma}{ }^{\delta} \boldsymbol{F}_{b \delta} \mathcal{S}\right. \\
& \left.+\left[\frac{2 \mathrm{i}}{3}\left(\gamma^{c}\right)^{\alpha \delta}\left(\nabla_{\alpha} \mathcal{S}\right)-\frac{\mathrm{i}}{2}\left(\gamma^{c}\right)_{\alpha \beta} C^{\alpha \beta \delta}\right] \boldsymbol{F}_{\gamma \delta}\right\} \\
+ & \frac{1}{6} E^{c} E^{b} E^{a} \varepsilon_{a b c}\left\{-\frac{1}{2} \varepsilon^{d e f} \nabla_{d} \boldsymbol{F}_{e f}+\left[\frac{\mathrm{i}}{2}\left(\gamma^{d}\right)_{\alpha \beta} C^{\alpha \beta \delta}-\frac{2 \mathrm{i}}{3}\left(\gamma^{d}\right)^{\alpha \delta}\left(\nabla_{\alpha} \mathcal{S}\right)\right] \boldsymbol{F}_{d \delta}\right\} . \quad(\mathrm{B} .2)
\end{aligned}
$$

The relation (B.2) provides us with a rule for "integration by parts" within the superform approach to constructing supersymmetric actions in three dimensions. Such an 
action is associated with a closed three-form, which plays the role of the Lagrangian. The action does not change if the Lagrangian is shifted by an exact three-form provided we are allowed to ignore boundary terms (in other words, all Lagrangians in the same cohomology class define the same action). In carrying out the explicit calculations for this paper, we have found extremely useful the following two special cases of the rule for "integration by parts" (B.2).

Firstly, given an antisymmetric tensor $\boldsymbol{F}_{a b}$, which is equivalent to the vector $\boldsymbol{F}^{a}=$ $\frac{1}{2} \varepsilon^{a b c} \boldsymbol{F}_{b c}$, it follows from $\mathrm{B} .2$ that

$$
\begin{aligned}
\frac{1}{2} E^{\gamma} E^{\beta} E^{a}\left\{\varepsilon_{a b c}\left(\gamma^{b}\right)_{\beta \gamma} \boldsymbol{F}^{c}\right\}= & \frac{1}{2} E^{\gamma} E^{b} E^{a} \varepsilon_{a b c}\left\{\frac{\mathrm{i}}{2} \nabla_{\gamma} \boldsymbol{F}^{c}\right\}+\frac{1}{6} E^{c} E^{b} E^{a} \varepsilon_{a b c}\left\{\frac{\mathrm{i}}{2} \nabla_{d} \boldsymbol{F}^{d}\right\} \\
& + \text { exact three-form . }
\end{aligned}
$$

Secondly, suppose that $\boldsymbol{F}_{a \beta}=\left(\gamma_{a}\right)_{\beta \gamma} \boldsymbol{V}^{\gamma}$, for some spinor $\boldsymbol{V}^{\gamma}$. Then it follows from (B.2) and (B.3) that

$$
\begin{aligned}
\frac{1}{2} E^{\gamma} E^{\beta} E^{a}\left\{\left(\gamma_{a}\right)_{\beta \gamma} \nabla^{\alpha} \boldsymbol{V}_{\alpha}\right\}= & \frac{1}{2} E^{\gamma} E^{b} E^{a} \varepsilon_{a b c}\{ \\
+ & -\frac{3}{2}(1+\lambda)\left(\gamma^{c}\right)_{\gamma}{ }^{\alpha} \boldsymbol{V}_{\alpha} \mathcal{S}-\frac{\mathrm{i}}{4}\left(\gamma^{c}\right)_{\gamma}{ }^{\alpha} \nabla^{2} \boldsymbol{V}_{\alpha} \\
& \left.+\frac{1}{2}\left(\gamma_{d}\right)_{\gamma}{ }^{\alpha} \nabla_{e} \boldsymbol{V}_{\alpha}-\frac{1}{2} \nabla^{c} \boldsymbol{V}_{\gamma}\right\} \\
& +\frac{1}{6} E^{c} E^{b} E^{a} \varepsilon_{a b c}\left\{2 \mathrm{i}\left(\nabla^{\alpha} \mathcal{S}\right) \boldsymbol{V}_{\alpha}-\frac{\mathrm{i}}{2}\left(\gamma^{d}\right)^{\rho \tau} \nabla_{d} \nabla_{\rho} \boldsymbol{V}_{\tau}\right\} \\
& + \text { exact three-form } .
\end{aligned}
$$

\section{References}

[1] S. Deser and J. H. Kay, "Topologically massive supergravity," Phys. Lett. B 120, 97 (1983).

[2] S. Deser, "Cosmological topological supergravity," in Quantum Theory Of Gravity, S. M. Christensen (Ed.), Adam Hilger, Bristol, 1984, pp. 374-381.

[3] P. van Nieuwenhuizen, "D = 3 conformal supergravity and Chern-Simons terms," Phys. Rev. D 32, $872(1985)$.

[4] M. Roček and P. van Nieuwenhuizen, " $N \geq 2$ supersymmetric Chern-Simons terms as $\mathrm{D}=3$ extended conformal supergravity," Class. Quant. Grav. 3, 43 (1986).

[5] U. Lindström and M. Roček, "Superconformal gravity in three dimensions as a gauge theory," Phys. Rev. Lett. 62, 2905 (1989).

[6] S. J. Gates, Jr., M. T. Grisaru, M. Roček and W. Siegel, Superspace, or One Thousand and One Lessons in Supersymmetry, Front. Phys. 58, 1 (1983) arXiv:hep-th/0108200. 
[7] B. M. Zupnik and D. G. Pak, "Superfield formulation of the simplest three-dimensional gauge theories and conformal supergravities," Theor. Math. Phys. 77 (1988) 1070 [Teor. Mat. Fiz. 77 (1988) 97].

[8] B. M. Zupnik and D. G. Pak, "Differential and integral forms in supergauge theories and supergravity," Class. Quant. Grav. 6, 723 (1989).

[9] P. S. Howe, J. M. Izquierdo, G. Papadopoulos and P. K. Townsend, "New supergravities with central charges and Killing spinors in 2+1 dimensions," Nucl. Phys. B 467, 183 (1996) arXiv:hepth/9505032].

[10] S. M. Kuzenko, U. Lindström and G. Tartaglino-Mazzucchelli, "Off-shell supergravity-matter couplings in three dimensions," JHEP 1103, 120 (2011) [arXiv:1101.4013 [hep-th]].

[11] D. Butter, S. M. Kuzenko and J. Novak, "The linear multiplet and ectoplasm," JHEP 1209, 131 (2012) arXiv:1205.6981 [hep-th]].

[12] M. F. Hasler, "The three-form multiplet in N=2 superspace," Eur. Phys. J. C 1, 729 (1998) hepth/9606076.

[13] S. J. Gates, Jr., "Ectoplasm has no topology: The prelude," in Supersymmetries and Quantum Symmetries, J. Wess and E. A. Ivanov (Eds.), Springer, Berlin, 1999, p. 46, arXiv:hep-th/9709104; "Ectoplasm has no topology," Nucl. Phys. B 541, 615 (1999) arXiv:hep-th/9809056.

[14] S. J. Gates, Jr., M. T. Grisaru, M. E. Knutt-Wehlau and W. Siegel, "Component actions from curved superspace: Normal coordinates and ectoplasm," Phys. Lett. B 421, 203 (1998) hep-th/9711151.

[15] P. S. Howe and R. W. Tucker, "A locally supersymmetric and reparametrization invariant action for a spinning membrane," J. Phys. A 10, L155 (1977); "Local supersymmetry in (2+1) dimensions. 1. Supergravity and differential forms," J. Math. Phys. 19, 869 (1978); "Local supersymmetry in $(2+1)$ dimensions. 2. An action for a spinning membrane," J. Math. Phys. 19, 981 (1978).

[16] M. Brown and S. J. Gates Jr., "Superspace Bianchi identities and the supercovariant derivative," Annals Phys. 122, 443 (1979).

[17] T. Uematsu, "Structure of $\mathrm{N}=1$ conformal and Poincare supergravity in (1+1)-dimensions and (2+1)-dimensions," Z. Phys. C 29, 143 (1985); "Constraints and actions in two-dimensional and three-dimensional N=1 conformal supergravity," Z. Phys. C 32, 33 (1986).

[18] U. Lindström and M. Roček, "A super-Weyl-invariant spinning membrane," Phys. Lett. B 218, 207 (1989).

[19] S. M. Kuzenko, "Prepotentials for $\mathrm{N}=2$ conformal supergravity in three dimensions," JHEP 1212 (2012) 021 arXiv:1209.3894 [hep-th]].

[20] M. Becker, D. Constantin, S. J. Gates Jr., W. D. Linch III, W. Merrell and J. Phillips, "M-theory on Spin(7) manifolds, fluxes and 3D, N = 1 supergravity," Nucl. Phys. B 683, 67 (2004) arXiv:hepth/0312040].

[21] N. Dragon, "Torsion and curvature in extended supergravity," Z. Phys. C 2, 29 (1979).

[22] J. Novak, "Superform formulation for vector-tensor multiplets in conformal supergravity," JHEP 1209, 060 (2012) arXiv:1205.6881 [hep-th]]. 